\title{
SIMPLIFYING STABLE MAPPINGS INTO THE PLANE FROM A GLOBAL VIEWPOINT
}

\author{
MAHITO KOBAYASHI AND OSAMU SAEKI
}

\begin{abstract}
Let $f: M \rightarrow \mathbf{R}^{2}$ be a $C^{\infty}$ stable map of an $n$-dimensional manifold into the plane. The main purpose of this paper is to define a global surgery operation on $f$ which simplifies the configuration of the critical value set and which does not change the diffeomorphism type of the source manifold $M$. For this purpose, we also study the quotient space $W_{f}$ of $f$, which is the space of the connected components of the fibers of $f$, and we completely determine its local structure for arbitrary dimension $n$ of the source manifold $M$. This is a completion of the result of Kushner, Levine and Porto for dimension 3 and that of Furuya for orientable manifolds of dimension 4. We also pay special attention to dimension 4 and obtain a simplification theorem for stable maps whose regular fiber is a torus or a 2 -sphere, which is a refinement of a result of Kobayashi.
\end{abstract}

\section{INTRODUCTION}

Let $f: M \rightarrow \mathbf{R}^{2}$ be a proper stable map of an $n$-dimensional manifold into the plane $(n \geq 3)$. It is often an important problem to change $f$ by some process to obtain another stable map $g: M \rightarrow \mathbf{R}^{2}$ which is simpler than the original stable map $f$. For example, Levine $[\mathbf{L} \mathbf{1}]$ has shown that, if $M$ is orientable, we can change $f$ by a homotopy so that the new map $g$ has at most one cusp point. In this paper, we will define a new surgery operation, called an $R$-operation, which converts a given stable map to another one which is simpler than the original one in the sense that the configuration of the critical value set is simpler. This is motivated by the work of the first author [Kob1], who defined two surgery operations on certain stable maps of simply connected 4-manifolds. Unfortunately, one of the operations he defined, called an $S$-operation, does not preserve the diffeomorphism type of the source manifold in general, although it preserves the homeomorphism type. Our $R$-operation is defined in a totally different manner, but has the same effect as an $S$-operation without changing the diffeomorphism type of the source manifold. Furthermore, our $R$-operation is also defined for certain higher dimensions.

In order to define an $R$-operation in a general context, we will need to define a pseudo quotient space $W$ and a pseudo quotient map $q: M \rightarrow W$, which are generalizations of the quotient space $W_{f}$ and the quotient map $q_{f}: M \rightarrow W_{f}$ of a stable map $f: M \rightarrow \mathbf{R}^{2}$ respectively (see, for example, [BdR, L2, KLP, Sa2]).

Received by the editors October 24, 1994.

1991 Mathematics Subject Classification. Primary 57R45; Secondary 57R35, 57M99.

The second author has been partially supported by CNPq, Brazil, and by Grant-in-Aid for Encouragement of Young Scientists (No. 07740063), Ministry of Education, Science and Culture, Japan. 
For this reason, we need to completely determine the local structure of the quotient space $W_{f}$ of a stable map $f$ into the plane. This will be done in $\S 2$ (Theorem 2.2 and Corollary 2.12). Note that the local structure of a quotient space has already been determined by Kushner, Levine and Porto [KLP, L2] for dimension 3 and by Furuya $[\mathbf{F u}, \mathbf{P F}]$ for the orientable case of dimension 4. Our result is a completion of their results for arbitrary dimensions. In particular, we will see that the quotient space of a stable map into the plane is a locally finite polyhedron such that each point has a standard conic neighborhood. This is a fundamental result in itself, and it is important in studying the global topology of stable maps into the plane.

The paper is organized as follows. In $\S 2$, we study the topology of the quotient space of a stable map and we determine its local structure. In $\S 3$, we define pseudo quotient spaces and pseudo quotient maps, which are generalizations of quotient spaces and quotient maps of stable maps respectively. One of the advantages of this generalization is that, in this generalized category, we can define a surgery operation, called an $R$-operation, which is defined in $\S 4$. We also obtain our simplification theorem of mappings (Theorem 4.13) in $\S 4$. In $\S 5$, we consider the existence problem of an "immersion" into the plane of a given pseudo quotient space so that we can define the $R$-operation in the category of stable maps, not in the category of pseudo quotient maps. Finally in $\S 6$, we study stable maps of 4-dimensional manifolds and we obtain a refinement of a result of [Kob1]. We also obtain some important corollaries of this refinement on 4-dimensional topology. In $\S 7$, as an example which well illustrates our simplification theorem, we consider pseudo quotient maps with only spherical regular fibers and we completely determine the diffeomorphism type of the source manifold of such a map.

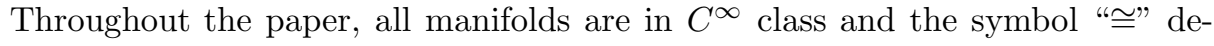
notes a diffeomorphism between manifolds or an appropriate isomorphism between algebraic objects.

\section{Local Structure of a QUOTIENT SPACE}

In this section, we study the topology of the quotient space of a stable map into the plane and we completely determine its local structure. Note that the case where the dimension of the source manifold is equal to 3 has already been studied by Kushner, Levine and Porto [KLP, L2], and the case where the source manifold is orientable of dimension 4, by Furuya $[\mathbf{F u}, \mathbf{P F}]$.

Let $f: M \rightarrow \mathbf{R}^{2}$ be a proper smooth map of an $n$-manifold into the plane. We denote the set of such maps by $C^{\infty}\left(M, \mathbf{R}^{2}\right)$, which is equipped with the Whitney $C^{\infty}$-topology. We say that $f$ is stable if the orbit of $f$ by the natural action of $A=$ $\operatorname{Diff}(M) \times \operatorname{Diff}\left(\mathbf{R}^{2}\right)$ is an open set of $C^{\infty}\left(M, \mathbf{R}^{2}\right)$. Note that the set of stable maps is open and dense in $C^{\infty}\left(M, \mathbf{R}^{2}\right)([\mathbf{M a}])$ and that the following characterization is known (see, for example, $[\mathbf{L 1}, \mathbf{L 2}, \mathbf{G G}]$ ). In the following, $S(f)$ denotes the singular set of $f$.

Proposition 2.1. Let $f: M \rightarrow \mathbf{R}^{2}$ be a proper smooth map. Then $f$ is stable if and only if the following local and global conditions are satisfied.

For every $p \in S(f)$, there exist local coordinates $\left(u, z_{1}, \cdots, z_{n-1}\right)$ centered at $p$ and $(U, Y)$ centered at $f(p)$ such that

$$
\left(L_{1}\right) \quad U \circ f=u, \quad Y \circ f=\sum_{i=1}^{n-1} \pm z_{i}^{2} \quad(p: \text { a fold point })
$$


or there exist local coordinates $\left(u, x, z_{1}, \cdots, z_{n-2}\right)$ centered at $p$ and $(U, Y)$ centered at $f(p)$ such that

$$
\left(L_{2}\right) \quad U \circ f=u, \quad Y \circ f=\sum_{i=1}^{n-2} \pm z_{i}^{2}+x u+x^{3} \quad(p: \text { a cusp point }),
$$

and

$\left(G_{1}\right) \quad$ for every cusp point $p \in S(f), f^{-1}(f(p)) \cap S(f)=\{p\} \quad$ and,

$\left.\left(G_{2}\right) \quad f\right|_{(S(f) \backslash\{\text { cusp points }\})}$ is an immersion with normal crossings.

We call a fold point $p$ a definite fold point if all the signs appearing in the summation in $\left(L_{1}\right)$ are the same. Otherwise, we call $p$ an indefinite fold point. We denote by $S_{0}(f)$ the set of the definite fold points of $f$. Note that if $f$ is stable, $S(f)$ is a closed regular 1-dimensional submanifold of $M$.

Now we recall the notion of the Stein factorization of a smooth map (see [BdR, KLP, L2]). Let $g: M \rightarrow N$ be a smooth map of an $n$-manifold into a $p$-manifold $(n>p)$. For $x, y \in M$, define $x \sim y$ if $g(x)=g(y)$ and $x, y$ are in the same connected component of $g^{-1}(g(x))=g^{-1}(g(y))$. We denote by $W_{g}$ the quotient space of $M$ by the equivalence relation $\sim$ and by $q_{g}: M \rightarrow W_{g}$ the quotient map. Note that there exists a unique map $\bar{g}: W_{g} \rightarrow N$ such that $g=\bar{g} \circ q_{g}$. The space $W_{g}$ or the commutative diagram

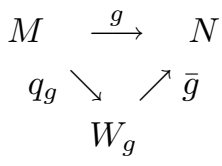

is called the Stein factorization of $g$.

The following theorem has been proved by Kushner, Levine and Porto [KLP, L2] for $n=3$ and by Furuya [Fu, PF] for $n=4$.

Theorem 2.2. Let $f: M \rightarrow \mathbf{R}^{2}$ be a proper stable map of an $n$-manifold into the plane $(n \geq 3)$. Then the quotient space $W_{f}$ is a Hausdorff space and every point $x \in W_{f}$ has a conic neighborhood as in Figure 1, where the thick lines indicate $q_{f}(S(f))$.

Remark 2.3. We can replace the target manifold $\mathbf{R}^{2}$ by arbitrary 2-dimensional manifolds and Theorem 2.2 still holds for this larger class of mappings. Note that stable mappings into 2-dimensional manifolds are defined in the same way as in the case of $\mathbf{R}^{2}$ and that Proposition 2.1 is also valid. In this paper, for simplicity, we almost always consider stable mappings into the plane.

Lemma 2.4. Let $f$ be as in Theorem 2.2. Then the quotient space $W_{f}$ of $f$ is a Hausdorff space.

Remark 2.5. The above lemma does not hold for non-proper maps in general. For example, consider the map $u=\pi \mid\left(\mathbf{R}^{2} \backslash\{0\}\right): \mathbf{R}^{2} \backslash\{0\} \rightarrow \mathbf{R}$, where $\pi: \mathbf{R}^{2} \rightarrow \mathbf{R}$ is the standard projection. Then the quotient space of $u$ is not a Hausdorff space. 
(a)

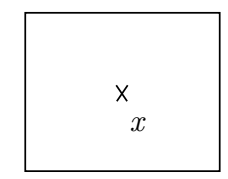

(b)

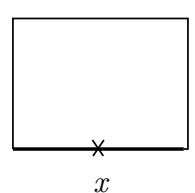

(b-1)

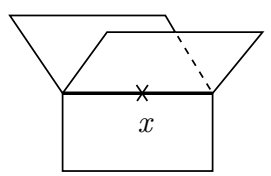

(b-2)

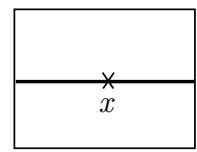

(b-3)

$x$ : boundary point

$x$ : branch point

(c)

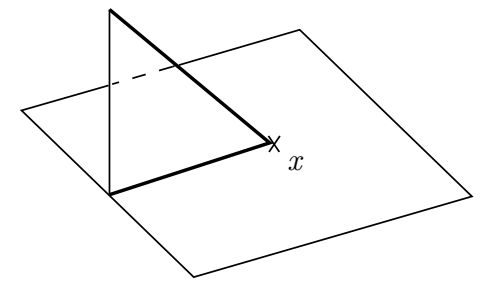

(c-1) cuspidal branch

(d)

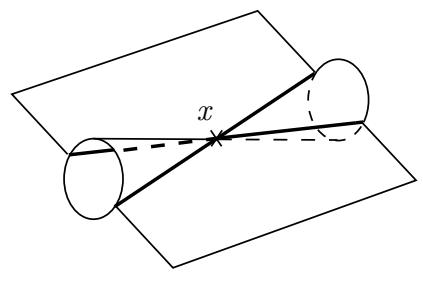

(d-1) double cone

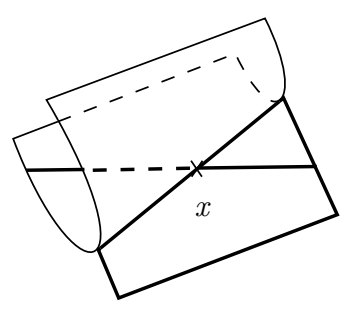

(d-3)
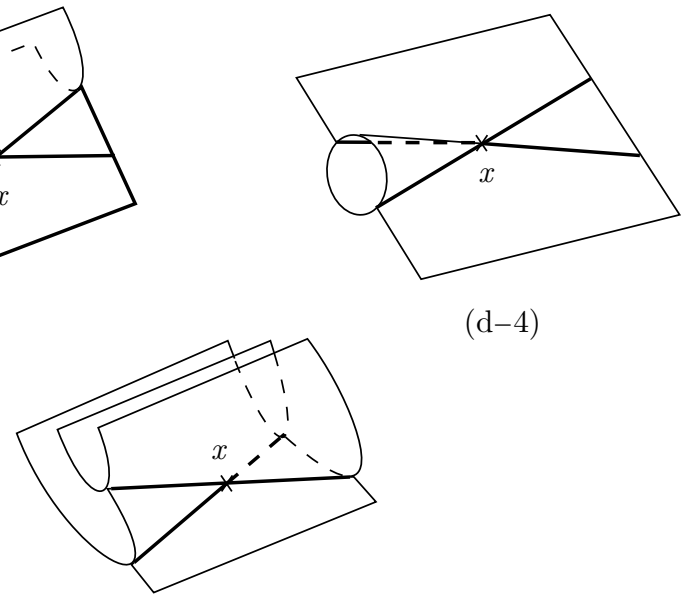

(d-4)

(d-5) trident

FiguRE 1 
Proof of Lemma 2.4. Take two distinct points $x, y \in W_{f}$. If $\bar{f}(x) \neq \bar{f}(y)$, then there exist disjoint open neighborhoods $U_{x}$ and $U_{y}$ of $\bar{f}(x)$ and $\bar{f}(y)$ respectively in $\mathbf{R}^{2}$. Set $V_{x}=\bar{f}^{-1}\left(U_{x}\right)$ and $V_{y}=\bar{f}^{-1}\left(U_{y}\right)$. Then we see that $V_{x}$ and $V_{y}$ are disjoint open sets of $W_{f}$ which separate $x$ and $y$. Thus we suppose $\bar{f}(x)=\bar{f}(y)=a \in \mathbf{R}^{2}$. The following lemma is proved in $[\mathbf{L 2}, \mathrm{p} .7]$ for $n=3$ and the same argument works also for general $n$. Hence we omit the proof here.

Lemma 2.6. Set $I=(-1,1)$ and $J=[-1,1]$. For each point $a \in f(M)$, there exists an embedding $\psi: I \times J \rightarrow \mathbf{R}^{2}$ such that $a=\psi(0,0)$ and that the composition

$$
h: f^{-1}(\psi(I \times J)) \stackrel{f}{\longrightarrow} \psi(I \times J) \stackrel{\psi^{-1}}{\longrightarrow} I \times J \stackrel{p_{1}}{\longrightarrow} I
$$

is a trivial bundle, where $p_{1}$ is the projection to the first factor.

Let $Z$ be the interior of $\psi(I \times J)$ and let $U_{x}$ and $U_{y}$ be the connected components of $f^{-1}(Z)$ containing $q_{f}^{-1}(x)$ and $q_{f}^{-1}(y)$ respectively. Note that $q_{f}\left(U_{x}\right)$ and $q_{f}\left(U_{y}\right)$ are open sets of $W_{f}$, since $q_{f}^{-1}\left(q_{f}\left(U_{x}\right)\right)=U_{x}$ and $q_{f}^{-1}\left(q_{f}\left(U_{y}\right)\right)=U_{y}$ are open sets of $M$. Recall that $q_{f}^{-1}(x) \cap q_{f}^{-1}(y)=\emptyset$, since $x \neq y$. For the proof of Lemma 2.4, it suffices to show that $U_{x} \cap U_{y}=\emptyset$.

Case 1. The case where $q_{f}^{-1}(x)$ contains a cusp point.

By the global condition $\left(G_{1}\right)$ of Proposition 2.1, $q_{f}^{-1}(y)$ contains no singular point of $f$, and hence $f \mid U_{y}: U_{y} \rightarrow Z$ is a submersion by the proof of Lemma 2.6. In particular, $U_{y}$ contains no cusp points, and hence $U_{x} \cap U_{y}=\emptyset$.

Case 2. The cases other than Case 1.

Set $T_{x}=\left(h \mid f^{-1}(Z)\right)^{-1}(0) \cap U_{x}$ and $T_{y}=\left(h \mid f^{-1}(Z)\right)^{-1}(0) \cap U_{y}$. Note that $T_{x}$ (resp. $T_{y}$ ) is connected since $U_{x}$ (resp. $U_{y}$ ) is diffeomorphic to $T_{x} \times(-1,1)$ (resp. $T_{y} \times(-1,1)$ ) by Lemma 2.6. Now suppose that a point $x_{1}$ in $q_{f}^{-1}(x)$ is connected by a path in $f^{-1}(Z)$ to a point $y_{1}$ in $q_{f}^{-1}(y)$. Since $h \mid f^{-1}(Z)$ is a trivial fibration, we see that $x_{1}$ and $y_{1}$ are connected by a path in $T_{0}=\left(h \mid f^{-1}(Z)\right)^{-1}(0)$. Since $Z$ does not contain the image of a cusp point, we see that the composition

$$
g_{1}: T_{0} \stackrel{f}{\longrightarrow} \psi(I \times \operatorname{Int} J) \stackrel{\psi^{-1}}{\longrightarrow} I \times \operatorname{Int} J \stackrel{p_{2}}{\longrightarrow} \operatorname{Int} J
$$

is a Morse function with at most two critical points and at most one critical value $c$, where $p_{2}$ is the projection to the second factor and $c=g_{1}\left(x_{1}\right)=g_{1}\left(y_{1}\right)$. Then, by an argument using a gradient like vector field $([\mathbf{M i}])$, we see that there exists a retraction $r: T_{0} \rightarrow g_{1}^{-1}(c)$. Thus we see that $x_{1}$ and $y_{1}$ are connected by a path in $g_{1}^{-1}(c)$. Since $g_{1}^{-1}(c) \subset f^{-1}(\bar{f}(x))$, this is a contradiction. This completes the proof. \|

Proof of Theorem 2.2. Take a point $x \in W_{f}$. Let $\psi: I \times J \rightarrow \mathbf{R}^{2}$ be an embedding for $a=\bar{f}(x)$ as in Lemma 2.6 and let $h$ be the map defined in the lemma. Let $T$ denote the connected component of $h^{-1}(0)$ containing $q_{f}^{-1}(x)$. Note that $T$ is a compact $(n-1)$-manifold with boundary. Since $h: f^{-1}(\psi(I \times J)) \rightarrow I$ is a trivial fibration, there exist an embedding $\phi: I \times T \rightarrow M$ and a smooth map $g: I \times T \rightarrow J$ such that $f \circ \phi=\psi \circ($ id $\times g): I \times T \rightarrow \mathbf{R}^{2}$, where $\phi(I \times T)$ is the connected component of $f^{-1}(\psi(I \times J))$ containing $q_{f}^{-1}(x)$ (see $[\mathbf{L 2}$, Definition 
(p. 9)]). Then by [L2, Lemma 2 (p.11)], there exists an embedding $\theta: W_{\mathrm{id} \times g} \rightarrow W_{f}$ such that the following diagram is commutative:

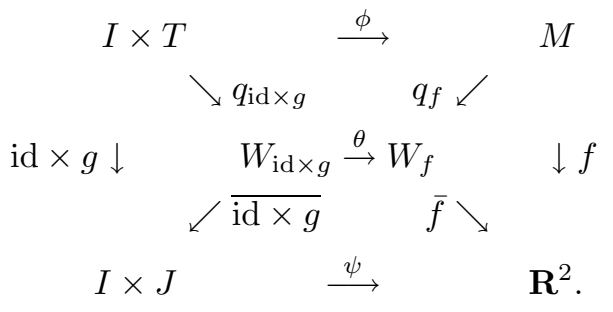

Note that we can prove the above lemma for every dimension $n$, by using the same argument as in [L2] for $n=3$. Note also that $U=\theta \circ q_{\mathrm{id} \times g}(I \times \operatorname{Int} T)$ is an open set of $W_{f}$, since $q_{f}^{-1}(U)$ is an open set $\operatorname{Int} \phi(I \times T)$ of $M$.

The following lemma is not difficult to prove.

Lemma 2.7. There exists an embedding $\eta: D^{2} \rightarrow \psi(I \times \operatorname{Int} J)$ such that $\eta(0)=$ $\bar{f}(x)$ and the composition

$$
\xi: f^{-1}\left(\eta\left(D^{2} \backslash\{0\}\right)\right) \stackrel{f}{\longrightarrow} \eta\left(D^{2} \backslash\{0\}\right) \stackrel{\eta^{-1}}{\longrightarrow} D^{2} \backslash\{0\} \stackrel{\alpha}{\longrightarrow} S^{1} \times(0,1] \stackrel{p_{2}}{\longrightarrow}(0,1]
$$

is a trivial bundle, where $\alpha$ is an appropriate diffeomorphism and $p_{2}$ is the projection to the second factor. In particular, there exists a diffeomorphism $\tau: f^{-1}\left(\eta\left(D^{2} \backslash\{0\}\right)\right)$ $\rightarrow f^{-1}\left(\eta\left(\partial D^{2}\right)\right) \times(0,1]$ such that the following diagram commutes:

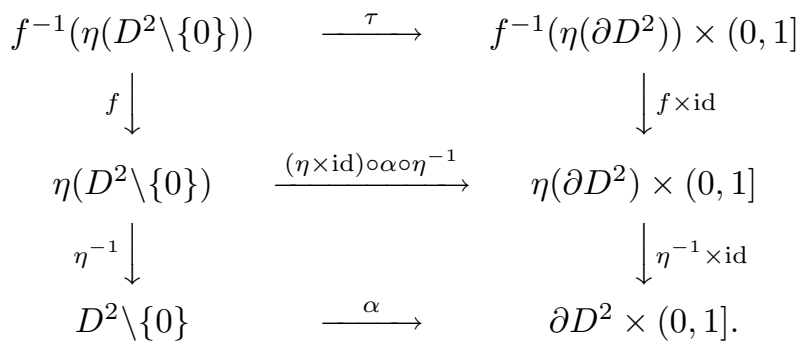

Set $S=\eta\left(\partial D^{2}\right)$ and $X=f^{-1}(S) \cap \phi(I \times T)$. Furthermore, set $k=f \mid X: X \rightarrow S$ and let $\tilde{S}$ be the connected component of $f^{-1}\left(\eta\left(D^{2}\right)\right)$ containing $q_{f}^{-1}(x)$. Note that $\tilde{S}=f^{-1}\left(\eta\left(D^{2}\right)\right) \cap \phi(I \times T)$ and that $\tau$ maps $\tilde{S} \backslash q_{f}^{-1}(x)$ diffeomorphically onto $X \times(0,1]$. Now define $\gamma: X \times[0,1] \rightarrow W_{f}$ by $\gamma(p, t)=q_{f}\left(\tau^{-1}(p, t)\right)$ if $t \neq 0$ and $\gamma(p, 0)=x(p \in X)$.

Lemma 2.8. $\gamma$ is continuous.

Proof. Take an open set $U$ of $W_{f}$ contained in $q_{f}(\phi(I \times T))$. If $x \notin U$, then it is easy to see that $\gamma^{-1}(U)$ is open in $X \times[0,1]$. If $x \in U$, then $\gamma^{-1}(U)=$ $(X \times\{0\}) \cup \tau\left(q_{f}^{-1}(U \backslash\{x\})\right)$. Since $q_{f}^{-1}(U)$ is an open neighborhood of $q_{f}^{-1}(x)$ in $\tilde{S}, \tau^{-1}(X \times(0, \varepsilon)) \cup q_{f}^{-1}(x) \subset q_{f}^{-1}(U)$ for a small positive real number $\varepsilon$. Thus $X \times(0, \varepsilon) \subset \tau\left(q_{f}^{-1}(U \backslash\{x\})\right)$, and hence $\gamma^{-1}(U)$ is open in $X \times[0,1]$. ॥ 
It is easy to see that $\gamma$ induces an injective continuous map $\bar{\gamma}: W_{k} \times[0,1] / W_{k} \times$ $\{0\} \rightarrow W_{f}$ such that the following diagram commutes:

$$
\begin{array}{cc}
X \times[0,1] & \\
q_{k} \times \operatorname{id} \downarrow & \searrow \gamma \\
W_{k} \times[0,1] & W_{f} \\
\pi \downarrow & \nearrow \bar{\gamma} \\
W_{k} \times[0,1] / W_{k} \times\{0\}, &
\end{array}
$$

where $\pi$ is the natural projection. Since $W_{k} \times[0,1] / W_{k} \times\{0\}$ is compact and $W_{f}$ is a Hausdorff space by Lemma $2.4, \bar{\gamma}$ is an embedding. Note that the image of $\bar{\gamma}$ is a neighborhood of $x$ in $W_{f}$. Thus we have proved the following.

Lemma 2.9. There exists a neighborhood of $x$ in $W_{f}$ which is homeomorphic to the cone over $W_{k}$, where $k: X \rightarrow S$ is the proper stable map of the $(n-1)$-manifold $X$ into $S\left(\cong S^{1}\right)$.

Note that Theorem 2.2 has been proved in $[\mathbf{L 2}]$ for $n=3$. Thus in the following, we assume that $n \geq 4$. Note that we do not assume that $M$ is orientable.

Lemma 2.10. There exists a point $a^{\prime} \in \eta\left(D^{2}\right) \backslash f(S(f))$ such that $f^{-1}\left(a^{\prime}\right) \cap \tilde{S}$ is connected.

Proof. If $q_{f}^{-1}(x) \cap S(f)=\emptyset$, then the result is obvious.

If $q_{f}^{-1}(x)$ contains a cusp, set $a^{\prime}=\psi(0, \varepsilon)$ for a sufficiently small non-zero real number $\varepsilon$. Near the cusp point, the composition

$$
f^{-1}(\psi(0 \times J)) \stackrel{f}{\longrightarrow} \psi(0 \times J) \stackrel{\psi^{-1}}{\longrightarrow} 0 \times J
$$

has the normal form

$$
\left(x, z_{1}, \cdots, z_{n-2}\right) \mapsto \sum_{i=1}^{n-2} \pm z_{i}^{2}+x^{3}
$$

(see $\left(L_{2}\right)$ of Proposition 2.1). Then it is not difficult to see that $f^{-1}\left(a^{\prime}\right) \cap \tilde{S}$ is connected.

Now suppose that $q_{f}^{-1}(x)$ contains a singular point which is not a cusp point. Then $q_{f}^{-1}(x)$ contains at most two singular points $p_{1}$ and $p_{2}$, which are fold singular points. For a point $b \in \mathbf{R}^{2}$ close to $\bar{f}(x), f^{-1}(b) \cap \tilde{S}$ and $q_{f}^{-1}(x)$ differ topologically only near $p_{1}$ and $p_{2}$. For $p_{1}$, there exists a "half disk region" $\Delta_{1}$ in $\eta\left(D^{2}\right)$ such that, for $b \in \Delta_{1} \backslash f(S(f)), f^{-1}(b) \cap \tilde{S}$ is "connected near $p_{1}$ ", since $n \geq 4$. We also have a similar half disk region $\Delta_{2}$ for $p_{2}$. Then a point $a^{\prime} \in \Delta_{1} \cap \Delta_{2} \backslash f(S(f))$ is a required point. II

Now we continue the proof of Theorem 2.2.

Case 1. $x \notin q_{f}(S(f))$.

In this case $k$ is just a smooth fiber bundle over $S$ with connected fibers. Thus $W_{k}$ is homeomorphic to $S^{1}$ and $x$ has a neighborhood as in Figure 1 (a). 


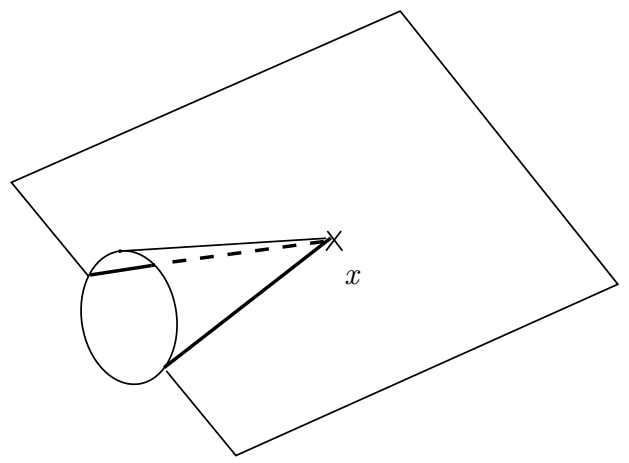

FiguRE 2

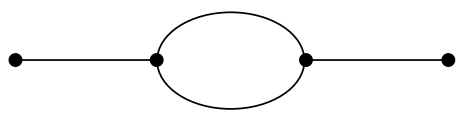

FiguRE 3

Case 2. $q_{f}^{-1}(x)$ contains exactly one singular point which is not a cusp point.

In this case $k$ has exactly two critical points. Then it is not difficult to see that $W_{k}$ is as in Figure $1(\mathrm{~b})$.

Case 3. $q_{f}^{-1}(x)$ contains a cusp point.

In this case $k$ has two critical points. By using the same arguments as in the proof of Lemma 2.10, we see easily that $W_{k}$ is as in Figure 1 (c) or as in Figure 2. Suppose that $W_{k}$ is as in Figure 2. Then for some $\delta \in I \backslash\{0\}$, the function

$$
l: f^{-1}(\psi(\delta \times J)) \cap \phi(I \times T) \stackrel{f}{\longrightarrow} \psi(\delta \times J) \stackrel{\psi^{-1}}{\longrightarrow} \delta \times J
$$

has exactly two critical points. Note that $l$ is a Morse function and its quotient space $W_{l}$ is homeomorphic to the space as in Figure 3. In particular $\pi_{1}\left(W_{l}\right) \cong \mathbf{Z}$ whose generator does not come from the regular fiber $l^{-1}(\delta \times 1)$ of $l$. On the other hand, by Lemma 2.6, $f^{-1}(\psi(\delta \times J)) \cap \phi(I \times T)$ is diffeomorphic to $f^{-1}(\psi(-\delta \times J)) \cap \phi(I \times T)$, which is diffeomorphic to $l^{-1}(\delta \times 1) \times J$, since the map corresponding to $l$ for $-\delta$ in place of $\delta$ has no critical point. Let $\pi_{+}: f^{-1}(\psi(\delta \times J)) \cap \phi(I \times T) \rightarrow l^{-1}(\delta \times 1)$ be the natural projection. Then we see that the induced homomorphism $\left(\pi_{+}\right)_{*}$ on the fundamental groups has kernel containing $\mathbf{Z}$ by the above observation. This is a contradiction. Thus $W_{k}$ is not as in Figure 2.

Case 4. $q_{f}^{-1}(x)$ contains two singular points of $f$.

Firstly note that the two singular points are indefinite fold points and that a small neighborhood of $\bar{f}(x)$ is divided into 4 regions by $C(f)=f(S(f))$. Note also that a regular fiber $f^{-1}(b)\left(b \in \mathbf{R}^{2} \backslash C(f)\right)$ of $f$ is a closed $(n-2)$-dimensional manifold and that, every time $b$ passes through $f\left(S(f) \backslash S_{0}(f)\right)$, the regular fiber changes by a surgery along an embedded $i$-sphere $(0 \leq i \leq n-3)$ in general. When $1 \leq i \leq n-4$, the number of components of the regular fiber is obviously unchanged. Denoting by $F$ the connected component $f^{-1}\left(a^{\prime}\right) \cap \tilde{S}$ of a regular fiber 
as in Lemma 2.10, we denote by $S_{1}$ and $S_{2}$ the two surgery spheres embedded in $F$ corresponding to the two singular points. If the dimension of $S_{1}$ and/or $S_{2}$ is equal to 0 , then we can choose another connected component of a regular fiber so that both of the surgery spheres have dimension greater than 0 . Thus we may assume that $\operatorname{dim} S_{1} \geq \operatorname{dim} S_{2} \geq 1$.

Firstly we consider the case where $\operatorname{dim} S_{1}=\operatorname{dim} S_{2}=n-3$. In this case, since the indices of the corresponding handles are equal, we may assume that $S_{1} \cap S_{2}=$ $\emptyset$. Furthermore $S_{1}$ and $S_{2}$ have trivial normal bundles $N\left(S_{1}\right)$ and $N\left(S_{2}\right)$ in $F$ respectively. We denote by $A_{j}$ and $B_{j}(j=1,2)$ the two connected components of $\partial N\left(S_{j}\right)$. The four connected sets $A_{1}, B_{1}, A_{2}$ and $B_{2}$ are divided into some classes by the equivalence relation defined by the existence of an arc in $F \backslash\left(\operatorname{Int} N\left(S_{1}\right) \cup\right.$ $\left.\operatorname{Int} N\left(S_{2}\right)\right)$ which connects given two sets. Since $F$ is connected, we may assume that $A_{1}$ and $A_{2}$ belong to the same class.

Case 4-1. When $A_{1}, A_{2}, B_{1}$ and $B_{2}$ all belong to the same class.

A surgery along $S_{1}$ and/or $S_{2}$ does not affect the connectivity of $F$. Hence, by Lemma 2.9, we see that a neighborhood of $x$ in $W_{f}$ is homeomorphic to the space as in Figure $1(\mathrm{~d}-2)$.

Case 4-2. When the four sets are divided into the two classes $\left\{A_{1}, A_{2}\right\}$ and $\left\{B_{1}, B_{2}\right\}$.

We see that a single surgery along $S_{1}$ or $S_{2}$ does not affect the connectivity of $F$. However, the successive surgeries along $S_{1}$ and $S_{2}$ make $F$ disconnected. Hence, we see that a neighborhood of $x$ in $W_{f}$ is homeomorphic to the space as in Figure $1(\mathrm{~d}-4)$.

Case 4-3. When the four sets are divided into the two classes $\left\{A_{1}, A_{2}, B_{1}\right\}$ and $\left\{B_{2}\right\}$.

By an argument similar to that in Case 4-2, we see that a neighborhood of $x$ in $W_{f}$ is homeomorphic to the space as in Figure $1(\mathrm{~d}-3)$.

Case 4-4. When the four sets are divided into the three classes $\left\{A_{1}, A_{2}\right\},\left\{B_{1}\right\}$ and $\left\{B_{2}\right\}$.

Similarly we see that a neighborhood of $x$ in $W_{f}$ is homeomorphic to the space as in Figure $1(\mathrm{~d}-5)$.

When $\operatorname{dim} S_{1}=n-3$ and $1 \leq \operatorname{dim} S_{2} \leq n-4$, the situation is the same as in Case 4-1 or 4-3 above. If both of the surgery spheres $S_{1}$ and $S_{2}$ have dimensions between 1 and $n-4$, the situation is as in Case 4-1.

Thus we have shown that a neighborhood of $x$ in $W_{f}$ must be homeomorphic to one of the spaces as in Figure 1 (d). This completes the proof of Theorem 2.2. II

For a future reference, we call the conic neighborhood as in Figure 1 (c-1) a cuspidal branch, $(\mathrm{d}-1)$ a double cone and $(\mathrm{d}-5)$ a trident. Note that the double cone does not occur for $n \geq 4$.

Remark 2.11. We do not know if a similar result holds also for stable maps into $\mathbf{R}^{p}$ with $n>p \geq 3$. If a result like Lemma 2.9 holds also in this case, we would be able to prove such a result by the induction on the target dimension $p$.

Theorem 2.2 has an immediate corollary as follows. 
Corollary 2.12. Let $f: M \rightarrow \mathbf{R}^{2}$ be a proper stable map of an $n$-manifold into the plane $(n \geq 3)$. Then $W_{f}$ is a locally finite polyhedron.

Remark 2.13. Note that all conic neighborhoods as in Figure 1 actually arise for some stable map. For example, see [ML, MPS].

Remark 2.14. Note that every conic neighborhood as in Figure 1 is equipped with a natural map into $\mathbf{R}^{2}$, which corresponds to the map $\bar{f}: W_{f} \rightarrow \mathbf{R}^{2}$ for a stable map $f: M \rightarrow \mathbf{R}^{2}$. We also note that every component of $W_{f} \backslash q_{f}(S(f))$ is canonically oriented by the local homeomorphism $\bar{f}$, once an orientation of $\mathbf{R}^{2}$ is fixed, and that their orientations are consistent along $q_{f}(S(f))$.

In the next section, we define generalizations of quotient spaces and quotient maps so that we have more freedom in changing a given map.

\section{Pseudo QUOTIENT MAPS AND CONFIGURATION TRIVIALITY}

In this section, we first define a generalization of quotient maps, namely pseudo quotient maps, and then we introduce a notion of configuration triviality of the critical value set of a pseudo quotient map. The advantage of this generalization is that we can define a surgery operation, which simplifies a given map in the generalized category, which will be seen in $\S 4$.

Definition 3.1. Let $W$ be a 2-dimensional locally finite polyhedron. We say that $W$ is a pseudo quotient space if every point $x$ of $W$ has a conic neighborhood as in Figure 1 in $\S 2$. Furthermore, we denote by $\Sigma(W)$ the set of the non-manifold points of $W$, including the boundary points.

A pseudo quotient space $W$ is said to be oriented if each connected component of $W \backslash \Sigma(W)$ is oriented and the orientations are consistent along $\Sigma(W)$ in the sense of Remark 2.14 .

The quotient space $W_{f}$ of a proper stable map $f: M \rightarrow \mathbf{R}^{2}$ of an $n$-manifold $M$ is always a pseudo quotient space in the above sense by Theorem 2.2 and Corollary 2.12. Furthermore, it is oriented, once an orientation of $\mathbf{R}^{2}$ is fixed. Furthermore, a branched surface as defined in $[\mathbf{S a 2}]$ is a pseudo quotient space in the above sense.

Definition 3.2. Let $q: M \rightarrow W$ be a proper continuous surjective map of an $n$-manifold into a pseudo quotient space $W(n \geq 3)$. We say that $q$ is a pseudo quotient map if for every point $x \in W$, there exist a conic neighborhood $U$ as in Figure 1 and a continuous map $\varphi: U \rightarrow \mathbf{R}^{2}$ as in Remark 2.14 such that $\psi=\varphi \circ q \mid q^{-1}(U): q^{-1}(U) \rightarrow \mathbf{R}^{2}$ is a proper stable map and that its quotient map is $C^{0}$-left equivalent to $q \mid q^{-1}(U)$; i.e., there exists a homeomorphism $\alpha: U \rightarrow W_{\psi}$ such that $\alpha^{-1} \circ q_{\psi}=q \mid q^{-1}(U)$.

In the above definition, the following diagram is commutative.

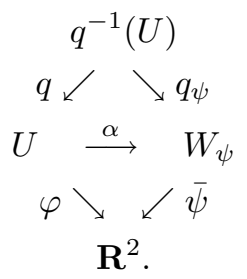


Note that the map $\varphi$ is finite-to-one, since $\psi$ is proper. Note also that the quotient map $q_{f}: M \rightarrow W_{f}$ of a proper stable map $f: M \rightarrow \mathbf{R}^{2}$ is always a pseudo quotient map.

For example, a fold map as defined in [Sa2] is a pseudo quotient map in the sense of Definition 3.2.

For a pseudo quotient map $q: M \rightarrow W$, we define its singularities as the union of the corresponding singularities of the stable maps $\varphi \circ q \mid q^{-1}(U)$ as in Definition 3.2. We denote by $S(q)$ the set of the singularities of $q$ and by $S_{0}(q)$ the set of the definite fold points. Furthermore, we set $C(q)=q(S(q))$, which is the set of the critical values of $q$. It is not difficult to see, with the help of the above commutative diagram, that $W \backslash C(q)$ admits a unique smooth structure so that $\varphi \mid(U \backslash C(q))$ is an immersion for every pair $(U, \varphi)$ as in Definition 3.2.

For a pseudo quotient map $q: M \rightarrow W$, if $S(q)=\emptyset$, then $W$ is a smooth nonsingular surface and $q$ is a smooth bundle projection. Thus, in this paper, we always assume that a pseudo quotient map has nonempty singular set.

In the next section we will introduce a geometric operation on pseudo quotient maps which simplifies the configuration of the critical value set in the following sense.

Definition 3.3. Let $q: M \rightarrow W$ be a pseudo quotient map of an $n$-manifold. A component $R$ of $W \backslash C(q)$ is said to be a spherical region if, for every point $x \in R$, $q^{-1}(x)$ is diffeomorphic to the standard sphere $S^{n-2}$. Note that if $\bar{R} \cap q\left(S_{0}(q)\right) \neq \emptyset$, i.e., if $R$ is adjacent to $q\left(S_{0}(q)\right)$, then $R$ is a spherical region.

Definition 3.4. We say that a pseudo quotient map $q: M \rightarrow W$ is configuration trivial with respect to the spherical regions if for every spherical region $R$ such that $\bar{R}$ is compact and that $\bar{R} \cap q\left(S_{0}(q)\right)=\emptyset, R$ is homeomorphic to the open 2-disk.

Remark 3.5. The above definition may seem a little strange. However, for stable maps of simply connected 4-manifolds studied in [Kob1], it coincides with the definition of the configuration triviality defined in [Kob1].

Lemma 3.6. Suppose that $q: M \rightarrow W$ is a pseudo quotient map of an n-manifold. If $q$ is configuration trivial with respect to the spherical regions and $W$ is connected, then $W \backslash\left(\bigcup_{i} R_{i}\right)$ is connected, where $R_{i}$ runs over the spherical regions $R$ of $W \backslash C(q)$ such that $\bar{R}$ is compact and that $\bar{R} \cap q\left(S_{0}(q)\right)=\emptyset$. Furthermore, if $H_{1}\left(M ; \mathbf{Z}_{2}\right)=$ 0 and $W \backslash\left(\bigcup_{i} R_{i}\right)$ is connected, then $q$ is configuration trivial with respect to the spherical regions.

Note that when $M$ is compact, the condition that $\bar{R}$ is compact is not necessary in Definition 3.4 and Lemma 3.6.

Proof of Lemma 3.6. Suppose that $q: M \rightarrow W$ is configuration trivial. Then each $R_{i}$ is homeomorphic to the open 2-disk. Now take two arbitrary points $z, w \in$ $W \backslash\left(\bigcup_{i} R_{i}\right)$. Then, since $W$ is connected, we can find a path $\alpha$ in $W$ which connects $z$ and $w$. By transversality on each $R_{i}$, we may assume that there exists a point $r_{i}$ in each $R_{i}$ which does not intersect $\alpha$. Then we can deform $\alpha$ on each $\bar{R}_{i} \backslash\left\{r_{i}\right\}$ fixing the end points of $\alpha$ so that $\bar{R}_{i} \cap \alpha \subset \bar{R}_{i} \backslash R_{i}$. This implies that $z$ and $w$ are connected by a path in $W \backslash\left(\bigcup_{i} R_{i}\right)$. This completes the first part of Lemma 3.6.

Suppose that $H_{1}\left(M ; \mathbf{Z}_{2}\right)=0$ and that $W \backslash\left(\bigcup_{i} R_{i}\right)$ is connected. Take a spherical region $R$ such that $\bar{R}$ is compact and that $\bar{R} \cap q\left(S_{0}(q)\right)=\emptyset$. There exists a compact 
surface $\tilde{R}$ with boundary whose interior is homeomorphic to $R$. (For example, $R \backslash \operatorname{Int} N$ is such a compact surface, where $N$ is a small regular neighborhood of $C(q)$ in $W$. Note that $\tilde{R}$ may not be homeomorphic to $\bar{R}$.) We will show that $\tilde{R}$ is homeomorphic to the 2-disk. Suppose that $\partial \tilde{R}$ is not connected. Then we have a properly embedded $\operatorname{arc} \alpha$ in $\tilde{R}$ and a simple closed curve $\beta$ in $\operatorname{Int} \tilde{R}$ which intersect each other transversely in one point. Let $\alpha^{\prime}$ and $\beta^{\prime}$ be the corresponding curves in $\bar{R}$. Since $W \backslash\left(\bigcup_{i} R_{i}\right)$ is connected, we can connect the end points of $\alpha^{\prime}$ in $W \backslash\left(\bigcup_{i} R_{i}\right)$ by a curve $\alpha_{1}^{\prime}$. Let $\alpha^{\prime \prime}$ be the closed curve in $W$ which is the union of $\alpha^{\prime}$ and $\alpha_{1}^{\prime}$. Set $V=q^{-1}\left(\beta^{\prime}\right)$, which is a compact codimension-1 submanifold of $M$. Then it is not difficult to see that we have a smooth closed curve $\tilde{\alpha}$ in $M$ such that $q(\tilde{\alpha})=\alpha^{\prime \prime}$ and that $\tilde{\alpha}$ and $V$ intersect each other transversely in one point, by modifying $\alpha_{1}^{\prime}$ if necessary. This implies that

$$
[V] \cdot[\tilde{\alpha}] \neq 0,
$$

where $[V] \in H_{n-1}\left(M ; \mathbf{Z}_{2}\right)$ and $[\tilde{\alpha}] \in H_{1}\left(M ; \mathbf{Z}_{2}\right)$ are the homology classes represented by $V$ and $\tilde{\alpha}$ respectively and $[V] \cdot[\tilde{\alpha}] \in \mathbf{Z}_{2}$ denotes the intersection number. This contradicts the assumption that $H_{1}\left(M ; \mathbf{Z}_{2}\right)=0$. Hence $\partial \tilde{R}$ should be connected. Next suppose that $\tilde{R}$ is not homeomorphic to the 2-disk. Then we have two simple closed curves $\gamma$ and $\delta$ in $R$ which intersect each other transversely in one point. Then an argument similar to the above one leads to a contradiction. Hence $\tilde{R}$ is homeomorphic to the 2-disk. This completes the proof. \|

In the following sections, we will be mainly interested in the case where $M$ is simply connected. In this case, by the above lemma, the configuration triviality is equivalent to the connectedness of the complement of certain spherical regions.

For two pseudo quotient maps $q_{i}: M_{i} \rightarrow W_{i}(i=1,2)$ such that $M_{i}$ and $W_{i}$ are possibly oriented, that $n=\operatorname{dim} M_{1}=\operatorname{dim} M_{2}$ and that $S_{0}\left(q_{1}\right) \neq \emptyset \neq S_{0}\left(q_{2}\right)$, we define a new pseudo quotient map $q_{1} \sharp q_{2}: M_{1} \sharp M_{2} \rightarrow W_{1} \sharp W_{2}$, called the connected sum of $q_{1}$ and $q_{2}$, as follows, where " $\sharp "$ means the connected sum and "घ" the boundary connected sum. Let $a_{i}(i=1,2)$ be a point in $\partial W_{i}=q_{i}\left(S_{0}\left(q_{i}\right)\right)$ and $U_{i}$ its small regular neighborhood in $W_{i}$. Let $\lambda_{i}(i=1,2)$ be the closure of $\partial U_{i} \backslash \partial W_{i}$, which is an arc joining two points in $\partial W_{i}$. Note that, when $W_{i}$ is oriented, $\lambda_{i}$ is naturally oriented as a part of the boundary of $\overline{W_{i} \backslash U_{i}}$. We take $U_{i}$ so that $\lambda_{i}$ is normal to $\partial W_{i}$. It is not difficult to see that $q_{i}^{-1}\left(U_{i}\right)$ is diffeomorphic to the $n$-disk and that $q_{i}^{-1}\left(\lambda_{i}\right)=\partial\left(q_{i}^{-1}\left(U_{i}\right)\right)$. Let $\varphi: \partial\left(\overline{M_{2} \backslash q_{2}^{-1}\left(U_{2}\right)}\right) \rightarrow \partial\left(\overline{M_{1} \backslash q_{1}^{-1}\left(U_{1}\right)}\right)$ and $\psi: \lambda_{2} \rightarrow \lambda_{1}$ be (orientation reversing) diffeomorphisms satisfying the equation $\left(q_{1} \mid q_{1}^{-1}\left(\lambda_{1}\right)\right) \circ \varphi=$ $\psi \circ\left(q_{2} \mid q_{2}^{-1}\left(\lambda_{2}\right)\right)$ on $q_{2}^{-1}\left(\lambda_{2}\right)$. Refer to [Kob1, p. 336] for the existence of such diffeomorphisms $\varphi$ and $\psi$. Set $M_{1} \sharp M_{2}=\overline{\left(M_{1} \backslash q_{1}^{-1}\left(U_{1}\right)\right)} \cup_{\varphi} \overline{\left(M_{2} \backslash q_{2}^{-1}\left(U_{2}\right)\right)}$ and $W_{1} \natural_{a_{1}, a_{2}} W_{2}=\overline{\left(W_{1} \backslash U_{1}\right)} \cup_{\psi} \overline{\left(W_{2} \backslash U_{2}\right)}$. Now we define

$$
q_{1} \sharp a_{1}, a_{2} q_{2}: M_{1} \sharp M_{2} \rightarrow W_{1} \natural_{a_{1}, a_{2}} W_{2}
$$

by

$$
\left(q_{1} \sharp_{a_{1}, a_{2}} q_{2}\right)\left|\overline{\left(M_{i} \backslash q_{i}^{-1}\left(U_{i}\right)\right)}=q_{i}\right| \overline{\left(M_{i} \backslash q_{i}^{-1}\left(U_{i}\right)\right)} \quad(i=1,2) .
$$

The map $q_{1} \sharp_{a_{1}, a_{2}} q_{2}$ is obviously a pseudo quotient map. It is also obvious from the construction that if $b_{i}(i=1,2)$ is a point in the same connected component of 
$\partial W_{i}$ as $a_{i}$, then $q_{1} \sharp_{b_{1}, b_{2}} q_{2}$ is right-left equivalent to $q_{1} \sharp a_{1}, a_{2} q_{2}$. Following [Kob1], we use the notation

$$
\left(M_{1} \sharp M_{2}, q_{1} \sharp a_{1}, a_{2} q_{2}\right)=\left(M_{1}, q_{1}\right) \bigsqcup_{a_{1}, a_{2}}\left(M_{2}, q_{2}\right) .
$$

We often omit the subscripts $a_{1}$ and $a_{2}$ if there is no confusion, for instance, in the case that $W_{1}$ and $W_{2}$ have connected boundaries. Note that the above operation is commutative and associative up to right-left equivalence.

In the next section, we will define a global surgery operation, called an $R$ operation, on a pseudo quotient map, which simplifies the configuration of the critical value set. Unfortunately this operation can be defined only for certain dimensions $n$ of the source manifold. In order to describe this restriction, we prepare some notations. For the standard $(n-2)$-sphere $S^{n-2}, \operatorname{Diff}^{+}\left(S^{n-2}\right)$ denotes the group of the orientation preserving diffeomorphisms of $S^{n-2}$, and $\pi_{0}\left(\operatorname{Diff}^{+}\left(S^{n-2}\right)\right.$ ) denotes its identity component. It is known that $\pi_{0}\left(\operatorname{Diff}^{+}\left(S^{n-2}\right)\right)$ is isomorphic to the group of homotopy $(n-1)$-spheres $\Theta_{n-1}([\mathbf{K M}, \mathbf{C 2}])$ for $n \geq 7$ and that $\pi_{0}\left(\operatorname{Difff}^{+}\left(S^{n-2}\right)\right)=0$ for $n=2,3,4,5,7,13, \cdots([\mathbf{C 1}, \mathbf{S m}, \mathbf{H}])$. The $R$-operation mentioned above will be defined for $n \geq 4$ with $\pi_{0}\left(\operatorname{Diff}^{+}\left(S^{n-2}\right)\right)=0$ in the next section.

The following lemma will be helpful for getting a geometric interpretation of the configuration triviality.

Lemma 3.7. Suppose that $\pi_{0}\left(\operatorname{Diff}^{+}\left(S^{n-2}\right)\right)=0$ and $n \geq 3$. A pseudo quotient map $q: M \rightarrow W$ of a closed connected n-manifold $M$ with $H_{1}\left(M ; \mathbf{Z}_{2}\right)=0$ is configuration trivial with respect to the spherical regions if and only if there exists a decomposition of the form

$$
(M, q)=\left(M_{1}, q_{1}\right) \natural\left(M_{2}, q_{2}\right) \downarrow \cdots \natural\left(M_{k}, q_{k}\right),
$$

where each $q_{i}: M_{i} \rightarrow W_{i}$ is a pseudo quotient map such that each spherical region not adjacent to $q_{i}\left(S_{0}\left(q_{i}\right)\right)$ is homeomorphic to the open 2-disk and that each spherical region adjacent to $q_{i}\left(S_{0}\left(q_{i}\right)\right)$ is homeomorphic to the open annulus or the open 2-disk.

Proof. Firstly suppose that $q$ is configuration trivial with respect to the spherical regions. Take a spherical region $R$ of $W \backslash C(q)$. Since $W$ is compact in our case, $\bar{R}$ is always compact. If $\bar{R} \cap q\left(S_{0}(q)\right)=\emptyset$, then $R$ is homeomorphic to the open 2 -disk. If $\bar{R} \cap q\left(S_{0}(q)\right) \neq \emptyset$, then $R$ is an open planar surface by the proof of Lemma 3.6. Let $\tilde{R}$ be the compact surface with boundary whose interior is diffeomorphic to $R$. If $\partial \tilde{R}$ is connected, then $R=W \backslash q\left(S_{0}(q)\right)$, and we have easily the conclusion $\left(k=1\right.$ and $\left.(M, q)=\left(M_{1}, q_{1}\right)\right)$. If $\partial \tilde{R}$ has exactly two components, then $R$ is homeomorphic to the open annulus. If $\partial \tilde{R}$ has three or more components, let $C$ be a component of $\partial \tilde{R}$ which has a 1-dimensional intersection with $\bar{R} \cap q\left(S_{0}(q)\right)$. Then there exist properly embedded disjoint $\operatorname{arcs} \gamma_{1}, \cdots, \gamma_{l}$ in $\tilde{R}$ such that the end points of $\gamma_{i}$ are in $C \cap q\left(S_{0}(q)\right)$ and that the closures of each connected component of $\tilde{R} \backslash\left(\bigcup_{i=1}^{l} \gamma_{i}\right)$ is homeomorphic to the annulus. We may assume that $\gamma_{i}$ are normal to $\partial \tilde{R}$. Furthermore, we may assume that $\gamma_{i}$ are embedded in $\bar{R} \subset W$. Then $X_{i}=q^{-1}\left(\gamma_{i}\right)$ is a smooth closed $(n-1)$-dimensional manifold which admits a Morse function with exactly two critical points. Then, by our dimension assumption, we 
see that $X_{i}$ is diffeomorphic to the $(n-1)$-sphere. Furthermore, by an argument similar to the proof of Lemma 3.6, we see that each $\gamma_{i}$ separates $W$ and that each $X_{i}$ separates $M$. In other words, $M \backslash \bigcup_{i=1}^{l} X_{i}$ has $(l+1)$ connected components, whose closures we denote by $M_{1}^{\prime}, \cdots, M_{l+1}^{\prime}$. Note that each boundary component of $M_{j}^{\prime}$ is diffeomorphic to the $(n-1)$-sphere by our assumption on $n$. Then by a standard argument, we can construct pseudo quotient maps $q_{j}^{\prime}: \tilde{M}_{j}^{\prime} \rightarrow W_{j}^{\prime}$ such that $\tilde{M}_{j}^{\prime}$ is the closed manifold obtained by attaching $n$-disks to the boundaries of $M_{j}^{\prime}$ and that

$$
\left.(M, q)=\left(\tilde{M}_{1}^{\prime}, q_{1}^{\prime}\right) \downarrow \cdots \downarrow-\tilde{M}_{l+1}^{\prime}, q_{l+1}^{\prime}\right) .
$$

(For example, see [Sa1].) If we apply the same operation to all the spherical regions adjacent to $q\left(S_{0}(q)\right)$, then we obtain a desired decomposition.

Conversely, if $q$ has the decomposition as above, it is clear that $q$ is configuration trivial with respect to the spherical regions. This completes the proof. ॥

Remark 3.8. We note that the above decomposition lemma is valid for all $n \geq 3$, without the assumption on $\pi_{0}\left(\operatorname{Diff}^{+}\left(S^{n-2}\right)\right)$, in the $C^{0}$-category; i.e., $(M, q)$ is $C^{0}$ -

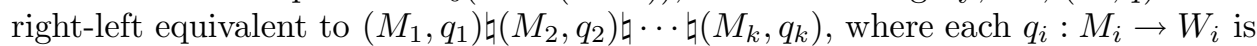
a " $C^{0}$ pseudo quotient map". In this case, $M_{i}$ is just a topological manifold and may not have a smooth structure.

\section{R-OPERATION}

In this section we introduce a surgery operation, called an $R$-operation, of pseudo quotient maps of compact manifolds, an operation which does not change the diffeomorphism type of the source manifold and which simplifies the configuration of the critical value set $C(q)$ of a given pseudo quotient map $q: M \rightarrow W$ in a global sense.

Let $q: M \rightarrow W$ be a pseudo quotient map of a closed connected $n$-manifold $(n \geq 3)$ into a pseudo quotient space $W$. We assume that $H_{1}\left(M ; \mathbf{Z}_{2}\right)=0$. Take a simple closed curve $c$ in a spherical region $R$.

Lemma 4.1. $W \backslash c$ has exactly two connected components.

Proof. Suppose that $W \backslash c$ is connected. Then we can find a simple closed curve in $M$ which intersects $q^{-1}(c)$ transversely in one point, as in the proof of Lemma 3.6. This contradicts the assumption on $H_{1}\left(M ; \mathbf{Z}_{2}\right)$. ॥

We denote by $A$ and $B$ the closures of the two connected components of $W \backslash c$.

Lemma 4.2. Suppose that $H_{1}(M ; \mathbf{Z})=0$ and $H_{2}(W ; \mathbf{Z})=0$. Then either $H_{1}(A ; \mathbf{Z})$ or $H_{1}(B ; \mathbf{Z})$ vanishes, and the other is isomorphic to the infinite cyclic group $\mathbf{Z}$.

For the proof of Lemma 4.2, we need the following.

Lemma 4.3. Suppose that $q: M \rightarrow W$ is a pseudo quotient map. Then the induced homomorphism $q_{*}: \pi_{1}(M) \rightarrow \pi_{1}(W)$ is surjective.

Proof. Take a loop $l$ in $W$. We may assume that $l$ does not pass through the image of the cusps and that $l \cap q\left(S_{0}(q)\right)=\emptyset$, by moving $l$ by a homotopy. Then it is easy to see that $l$ can be lifted to a loop in $M$. This completes the proof. I 
Proof of Lemma 4.2. By Lemma 4.3 and our assumption, we see that $H_{1}(W ; \mathbf{Z})=0$. Consider the following Mayer-Vietoris exact sequence with coefficients in $\mathbf{Z}$ :

$$
H_{2}(W) \rightarrow H_{1}(A \cap B) \rightarrow H_{1}(A) \oplus H_{1}(B) \rightarrow H_{1}(W) .
$$

Since $H_{1}(W)=0=H_{2}(W)$ and $A \cap B=c$, we see that $H_{1}(A) \oplus H_{1}(B) \cong \mathbf{Z}$. This completes the proof. $\|$

In what follows, we adopt the convention that $H_{1}(A ; \mathbf{Z})=0$ and hence that $H_{1}(B ; \mathbf{Z}) \cong \mathbf{Z}$. We also assume that $\pi_{0}\left(\operatorname{Diff}^{+}\left(S^{n-2}\right)\right)=0$ and $n \geq 3$. Set $\tilde{A}=q^{-1}(A)$ and $\tilde{B}=q^{-1}(B)$, which are codimension 0 submanifolds of $M$ with boundary. It is obvious that $M=\tilde{A} \cup \tilde{B}$. Furthermore $\tilde{A} \cap \tilde{B}=\partial \tilde{A}=\partial \tilde{B}$ and $q \mid \tilde{A} \cap \tilde{B}: \tilde{A} \cap \tilde{B} \rightarrow c$ is an orientable smooth fiber bundle with fiber $S^{n-2}$, since $c$ lies in a spherical region. Then, by our assumption on $n$, we see that it is a trivial bundle and that $\tilde{A} \cap \tilde{B}$ is diffeomorphic to $S^{1} \times S^{n-2}$.

Now we define two manifolds $M_{1}, M_{2}$ and two pseudo quotient maps $q_{1}, q_{2}$ of $M_{1}, M_{2}$ respectively as follows. Since $q \mid \partial \tilde{A}$ and hence $q \mid \partial \tilde{B}$ are the trivial $S^{n-2}$ bundles, there exist diffeomorphisms $\varphi_{i}$ and $\psi_{i}(i=1,2)$ which make the following diagrams commutative:
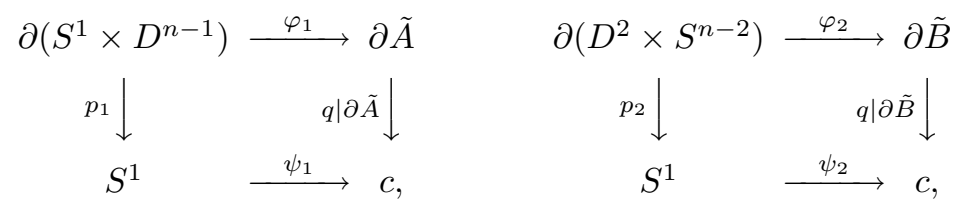

where $p_{1}$ and $p_{2}$ are the obvious projections. Let $g_{1}: S^{1} \times D^{n-1} \rightarrow S^{1} \times[0,1]$ be the map defined by $g_{1}(t, x)=\left(t,\|x\|^{2}\right)$, where $D^{n-1}$ is the unit disk in $\mathbf{R}^{n-1}$ and $\|x\|$ denotes the usual norm of $x \in \mathbf{R}^{n-1}$. Note that $g_{1}$ has definite fold points along $S^{1} \times\{0\} \subset S^{1} \times D^{n-1}$ and that $g_{1} \mid \partial\left(S^{1} \times D^{n-1}\right)=p_{1}: \partial\left(S^{1} \times D^{n-1}\right) \rightarrow S^{1} \times\{1\}$. Furthermore, let $g_{2}: D^{2} \times S^{n-2} \rightarrow D^{2}$ be the projection to the first factor. Set

$$
M_{1}=\tilde{A} \cup_{\varphi_{1}} S^{1} \times D^{n-1} \quad \text { and } \quad W_{1}=A \cup_{\psi_{1}} S^{1} \times[0,1]
$$

where $\psi_{1}$ is regarded as a diffeomorphism from $S^{1} \times 1$ to $c \subset A$. We define the pseudo quotient map $q_{1}: M_{1} \rightarrow W_{1}$ by

$$
q_{1}|\tilde{A}=q| \tilde{A} \quad \text { and } \quad q_{1} \mid\left(S^{1} \times D^{n-1}\right)=g_{1} .
$$

Furthermore, set

$$
M_{2}=\tilde{B} \cup_{\varphi_{2}} D^{2} \times S^{n-2} \quad \text { and } \quad W_{2}=B \cup_{\psi_{2}} D^{2} .
$$

We define the other pseudo quotient map $q_{2}: M_{2} \rightarrow W_{2}$ by

$$
q_{2}|\tilde{B}=q| \tilde{B} \quad \text { and } \quad q_{2} \mid\left(D^{2} \times S^{n-2}\right)=g_{2} .
$$


Definition 4.5. Let $q: M \rightarrow W$ be a pseudo quotient map of a closed connected $n$-manifold $M$. We assume that $\pi_{0}\left(\operatorname{Diff}^{+}\left(S^{n-2}\right)\right)=0, n \geq 3, H_{1}(M ; \mathbf{Z})=0$ and $H_{2}(W ; \mathbf{Z})=0$. Let $q_{1}: M_{1} \rightarrow W_{1}$ and $q_{2}: M_{2} \rightarrow W_{2}$ be the pseudo quotient maps which were constructed in the previous paragraph. We also assume that $S_{0}\left(q_{1}\right)$ and $S_{0}\left(q_{2}\right)$ are both non-empty. Then, an $R$-operation is the procedure which converts $q$ to the pseudo quotient map $q_{1} \sharp_{a, b} q_{2}: M_{1} \sharp M_{2} \rightarrow W_{1} \natural_{a, b} W_{2}$ for some points $a \in \partial W_{1}=q_{1}\left(S_{0}\left(q_{1}\right)\right)$ and $b \in \partial W_{2}=q_{2}\left(S_{0}\left(q_{2}\right)\right)$. For the change of the target pseudo quotient space and the configuration of the critical value set, see Figure 4 as an example.

We note that the reverse operation of an $R$-operation can also be defined.

Remark 4.6. When $\pi_{0}\left(\operatorname{Diff}^{+}\left(S^{n-2}\right)\right) \neq 0$, we cannot define an $R$-operation in the $C^{\infty}$-category. However, it is known that $\pi_{0}\left(\operatorname{Homeo}^{+}\left(S^{n-2}\right)\right)=0$ for all $n \geq 2$, where $\mathrm{Homeo}^{+}\left(S^{n-2}\right)$ is the group of the orientation preserving homeomorphisms of $S^{n-2}$. By using this, we can still define an $R$-operation in the $C^{0}$-category; i.e., in a situation as in Definition 4.5, without the assumption on $n$, we can find topological manifolds $M_{i}$ and " $C^{0}$ pseudo quotient maps" $q_{i}: M_{i} \rightarrow W_{i}(i=1,2)$ and we can construct a new $C^{0}$ pseudo quotient map $q_{1} \sharp_{a, b} q_{2}: M_{1} \sharp M_{2} \rightarrow W_{1} \bigsqcup_{a, b} W_{2}$.

Note that an $R$-operation preserves the diffeomorphism type of the source manifold provided that it is simply connected, which will be verified by the lemmas below.

Lemma 4.7. Assume that $\pi_{1}(M)=1$ and that $n=\operatorname{dim} M \geq 4$. Then an $R$ operation performed on a pseudo quotient map $q: M \rightarrow W$ as in Definition 4.5 preserves the diffeomorphism type of $M$; namely $M \cong M_{1} \sharp M_{2}$.

To show the above lemma we need the following.

Lemma 4.8. If $\pi_{1}(M)=1$ and $n=\operatorname{dim} M \geq 4$, then $\pi_{1}(\tilde{A})=1$.

Proof. In the following, we consider homology groups with $\mathbf{Z}$-coefficients. Firstly consider the Meyer-Vietoris exact sequence:

$$
H_{1}(\tilde{A} \cap \tilde{B}) \stackrel{\left(j_{1}\right)_{*} \stackrel{\oplus\left(j_{2}\right)_{*}}{\longrightarrow}}{\longrightarrow} H_{1}(\tilde{A}) \oplus H_{1}(\tilde{B}) \rightarrow H_{1}(M),
$$

where $j_{1}: \tilde{A} \cap \tilde{B} \rightarrow \tilde{A}$ and $j_{2}: \tilde{A} \cap \tilde{B} \rightarrow \tilde{B}$ are the inclusion maps and $H_{1}(M)=0$ by our assumption. Furthermore we have the commutative diagram

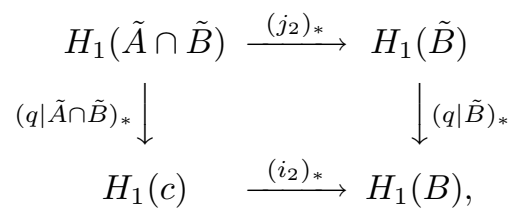

where $i_{1}: c \rightarrow A$ and $i_{2}: c \rightarrow B$ are the inclusion maps, $(q \mid \tilde{A} \cap \tilde{B})_{*}$ is an isomorphism and $\left(i_{2}\right)_{*}$ is also an isomorphism, by the proof of Lemma 4.2. Together with the exact sequence (4.9), this implies $H_{1}(\tilde{A})=0, H_{1}(\tilde{B}) \cong \mathbf{Z}$ and $\left(j_{2}\right)_{*}: H_{1}(\tilde{A} \cap \tilde{B}) \rightarrow H_{1}(\tilde{B})$ is an isomorphism. Consider the commutative diagram

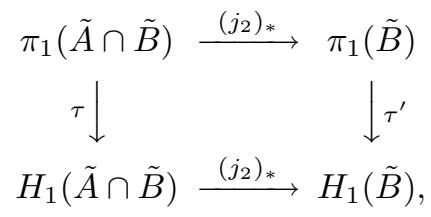


SIMPLIFYING STABLE MAPPINGS INTO THE PLANE

2623
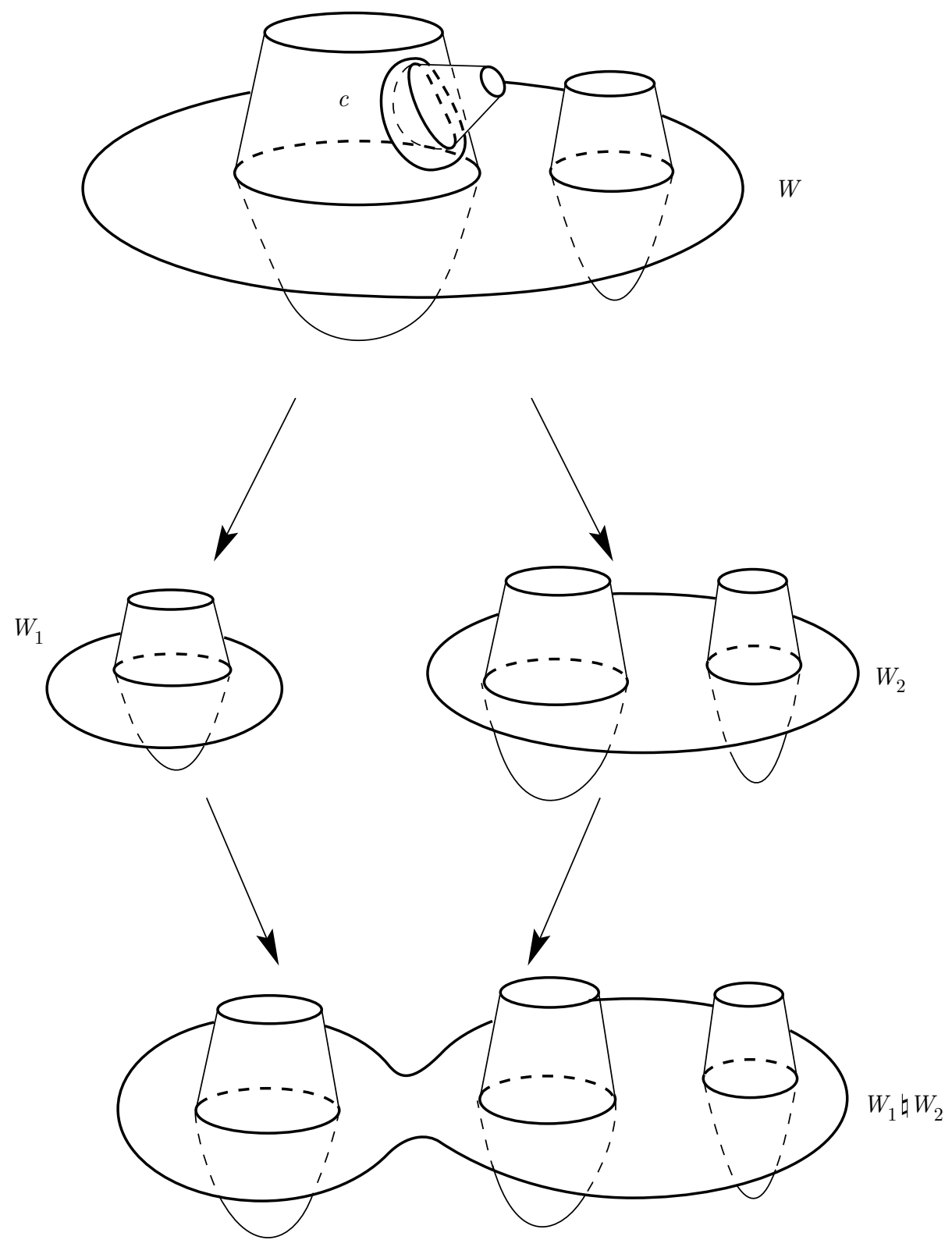

FIGURE 4

where $\tau$ and $\tau^{\prime}$ are the abelianizations. Since $\tau$ and the lower $\left(j_{2}\right)_{*}$ are isomerphisms, we have $\omega \circ\left(j_{2}\right)_{*}=\mathrm{id}_{\pi_{1}(\tilde{A} \cap \tilde{B})}$, where $\omega=\tau^{-1} \circ\left(j_{2}\right)_{*} \circ \tau^{\prime}: \pi_{1}(\tilde{B}) \rightarrow \pi_{1}(\tilde{A} \cap \tilde{B})$. Then, by using van Kampen theorem, we see that there exists a homomorphism $\xi: \pi_{1}(M) \stackrel{\cong}{\longrightarrow} \pi_{1}(\tilde{A}) *_{\pi_{1}(\tilde{A} \cap \tilde{B})} \pi_{1}(\tilde{B}) \rightarrow \pi_{1}(\tilde{A})$ such that $\xi \circ\left(k_{1}\right)_{*}=\mathrm{id}: \pi_{1}(\tilde{A}) \rightarrow$ $\pi_{1}(\tilde{A})$, where $k_{1}: \tilde{A} \rightarrow M$ is the inclusion map. Since $\pi_{1}(M)$ is trivial, this implies that $\pi_{1}(\tilde{A})=1$. $\|$ 
Remark 4.10. The above proof shows that $\pi_{1}(\tilde{B})$ is normally generated by $\left(j_{2}\right)_{*}(\alpha)$, where $\alpha \in \pi_{1}(\tilde{A} \cap \tilde{B}) \cong \mathbf{Z}$ is a generator. This implies that

$$
\pi_{1}\left(M_{2}\right)=\pi_{1}\left(\tilde{B} \cup_{\varphi_{2}} D^{2} \times S^{n-2}\right)=1 .
$$

Note also that $1=\pi_{1}(\tilde{A}) \cong \pi_{1}\left(\tilde{A} \cup_{\varphi_{1}} S^{1} \times D^{n-1}\right)=\pi_{1}\left(M_{1}\right)$.

Proof of Lemma 4.7. Firstly set $\varphi=\varphi_{1}^{-1} \circ \varphi_{2}: \partial\left(D^{2} \times S^{n-2}\right) \rightarrow \partial\left(S^{1} \times D^{n-1}\right)$, which is a diffeomorphism, where $\varphi_{1}$ and $\varphi_{2}$ are the diffeomorphisms as in diagram (4.4). Then it is easy to see that $\left(S^{1} \times D^{n-1}\right) \cup_{\varphi}\left(D^{2} \times S^{n-2}\right)$ is diffeomorphic to $S^{n}$.

By Lemma 4.8 and van Kampen theorem, we see that $M_{1}=\tilde{A} \cup_{\varphi_{1}} S^{1} \times D^{n-1}$ is simply connected. Set $K=S^{1} \times D^{n-1} \subset M_{1}$. Then, since $n=\operatorname{dim} M_{1} \geq 4$, we see that there exists an embedded $n$-disk $D$ in $M_{1}$ such that $K \subset \operatorname{Int} D$. Then we see that

$$
\begin{aligned}
\tilde{A} & =M_{1} \backslash \operatorname{Int} K \\
& =\left(M_{1} \backslash \operatorname{Int} D\right) \cup(D \backslash \operatorname{Int} K) \\
\cong & M_{1} \sharp\left(\left(D^{n} \cup D\right) \backslash \operatorname{Int} K\right) \\
& \cong M_{1} \sharp\left(S^{n} \backslash S^{1} \times D^{n-1}\right) \\
& \cong M_{1} \sharp D^{2} \times S^{n-2} .
\end{aligned}
$$

Here, $\partial \tilde{A}$ and $\partial\left(M_{1} \sharp D^{2} \times S^{n-2}\right)=\partial\left(D^{2} \times S^{n-2}\right)$ are identified by the diffeomorphism $\varphi^{-1} \circ \varphi_{1}^{-1}$. Hence we see that

$$
\begin{aligned}
M & =\tilde{A} \cup_{\text {id }} \tilde{B} \\
& \cong\left(M_{1} \sharp D^{2} \times S^{n-2}\right) \cup_{\varphi^{-1} \circ \varphi_{1}^{-1} \text { oid }} \tilde{B} \\
& \cong M_{1} \sharp\left(D^{2} \times S^{n-2} \cup_{\varphi_{2}^{-1}} \tilde{B}\right) \\
& =M_{1} \sharp M_{2} .
\end{aligned}
$$

This completes the proof of Lemma 4.7. ||

Recall that we have assumed that $H_{2}(W ; \mathbf{Z})=0$ in order to define an $R$-operation for a pseudo quotient map $q: M \rightarrow W$. Furthermore, if $\pi_{1}(M)=1$, then $\pi_{1}(W)=1$ by Lemma 4.3. Since $W$ is a 2-dimensional polyhedron, this implies that $W$ is contractible.

Lemma 4.11. Let $W_{1}$ and $W_{2}$ be the pseudo quotient spaces as in Definition 4.5. If $W$ is contractible, then so are $W_{1}$ and $W_{2}$.

Proof. In the following, we consider homology groups with $\mathbf{Z}$-coefficients. Consider the Mayer-Vietoris exact sequence:

$$
H_{2}(c) \rightarrow H_{2}(A) \oplus H_{2}(B) \rightarrow H_{2}(W) \rightarrow H_{1}(c) \rightarrow H_{1}(A) \oplus H_{1}(B) \rightarrow H_{1}(W) .
$$

Since $H_{2}(c), H_{2}(W)$ and $H_{1}(W)$ vanish, we see that $H_{2}(A), H_{2}(B)$ and $H_{1}(A)$ also vanish and that $H_{1}(B) \cong \mathbf{Z}$. Then, by the same argument as in the proof of Lemma 4.8 , we see that $\pi_{1}(A)=1$ and that $\pi_{1}(B)$ is normally generated by the class $[c] \in \pi_{1}(B)$. Then we see that

$$
W_{1}=A \cup_{\psi_{1}} S^{1} \times[0,1] \quad \text { and } \quad W_{2}=B \cup_{\psi_{2}} D^{2}
$$


are simply connected. Furthermore, since $W_{1}$ is homeomorphic to $A$, we have $H_{2}\left(W_{1}\right) \cong H_{2}(A)=0$, and by the Mayer-Vietoris exact sequence

$$
H_{2}(B) \oplus H_{2}\left(D^{2}\right) \rightarrow H_{2}\left(W_{2}\right) \rightarrow H_{1}(c) \rightarrow H_{1}(B) \oplus H_{1}\left(D^{2}\right),
$$

we also see that $H_{2}\left(W_{2}\right)=0$. Hence $W_{1}$ and $W_{2}$ are contractible. This completes the proof. II

The following lemma will be proved in the next section.

Lemma 4.12. Let $q: M \rightarrow W$ be a pseudo quotient map of a closed connected $n$-manifold into a compact pseudo quotient space such that

$$
H_{*}\left(W ; \mathbf{Z}_{2}\right) \cong H_{*}\left(\{\text { point }\} ; \mathbf{Z}_{2}\right)
$$

and that $W$ contains no trident (Figure $1(\mathrm{~d}-5)$ ). Then $W$ contains a boundary point (Figure $1(\mathrm{~b}-1)$ ).

Now we state the main theorem of this section as follows.

Theorem 4.13. Let $q: M \rightarrow W$ be a pseudo quotient map of a closed connected $n$ manifold such that $\pi_{0}\left(\operatorname{Diff}^{+}\left(S^{n-2}\right)\right)=0, n \geq 4, \pi_{1}(M)=1, H_{2}(W ; \mathbf{Z})=0$ and that $W$ contains no trident (Figure $1(\mathrm{~d}-5)$ ). Then, by a finite iteration of $R$-operations performed on $q$, we can convert $q$ to a pseudo quotient map $\tilde{q}: M \rightarrow \tilde{W}$ which is configuration trivial with respect to the spherical regions.

Proof. Take a spherical region $R$ of $W \backslash q\left(S_{0}(q)\right)$ such that $\bar{R} \cap q\left(S_{0}(q)\right)=\emptyset$. Firstly note that $R$ is an open planar surface by the proof of Lemma 3.6. Let $\tilde{R}$ be the compact surface with boundary whose interior is homeomorphic to $R$. In the following, we identify $R$ with $\operatorname{Int} \tilde{R}$. Furthermore, we define the number of boundary components of $R$ to be that of $\tilde{R}$. Now, if $R$ is not homeomorphic to the open 2-disk, take a simple closed curve $c$ in $R$ which is parallel to a component of $\partial \tilde{R}$. Then perform the $R$-operation along $c$ to obtain a new pseudo quotient map $q^{\prime}: M \rightarrow W^{\prime}$. Here we note that, by Lemma 4.11, the two target pseudo quotient spaces of the two pseudo quotient maps constructed just before Definition 4.5 for $q$ are contractible and contain no trident. Thus, by Lemma 4.12, we can perform the $R$-operation as desired. Note that, by this procedure, the number of spherical regions not adjacent to $q\left(S_{0}(q)\right)$ is unchanged and that the new spherical region not adjacent to $q^{\prime}\left(S_{0}\left(q^{\prime}\right)\right)$ corresponding to $R$ is homeomorphic to the open 2-disk or it has fewer boundary components than $R$. Furthermore, the new pseudo quotient map satisfies the conditions as in the theorem.

Since $M$ is compact, $W$ has a finite number of spherical regions. Thus, by performing the above operations finitely many times, we finally obtain a pseudo quotient map $\tilde{q}: M \rightarrow \tilde{W}$ such that each spherical region not adjacent to $\tilde{q}\left(S_{0}(\tilde{q})\right)$ is homeomorphic to the open 2 -disk, i.e., $\tilde{q}$ is configuration trivial with respect to the spherical regions. This completes the proof. ॥

Remark 4.14. By the proof of Theorem 4.13, $q$ and $\tilde{q}$ have the same number of spherical regions. Furthermore, the numbers of spherical regions not adjacent to the images of the definite fold points are also the same. 


\section{ImMERSING $\mathbf{Z}_{2}$-ACYCLIC PSEUdo QUOTIENT SPACES INTO $\mathbf{R}^{2}$}

Definition 5.1. Let $W$ be a pseudo quotient space. A proper continuous map $\eta: W \rightarrow \mathbf{R}^{2}$ is a topological immersion if, for each point $x \in W$, there exists a conic neighborhood as in Figure 1 on which $\eta$ is $C^{0}$ right-left equivalent to the canonical map into $\mathbf{R}^{2}$ as mentioned in Remark 2.14.

Let $q: M \rightarrow W$ be a pseudo quotient map of an $n$-manifold into a pseudo quotient space. We say that a proper topological immersion $\eta: W \rightarrow \mathbf{R}^{2}$ is a generic smooth immersion with respect to $q$ if the map $\eta \circ q: M \rightarrow \mathbf{R}^{2}$ is a $C^{\infty}$ stable map.

For a pseudo quotient space $W$, we denote by $B R(W)$ the set of the points in $W$ which have a conic neighborhood as in Figure 1 (b-2). We call a point in $B R(W)$ a branch point. It is easy to see that $B R(W)$ consists of some open arcs and circles. Note that for every branch point $x \in B R(W)$, its regular neighborhood $V_{x}$ is homeomorphic to the trivial $Y$-bundle over a closed interval, where $Y=\left\{r e^{2 \pi i \theta} \in\right.$ $\mathbf{C} ; 0 \leq r \leq 1, \theta=0,1 / 3,2 / 3\}$. Suppose that there exists a pseudo quotient map $q: M \rightarrow W$ of an $n$-manifold into $W$. Then, by the definition of a pseudo quotient map, we have a continuous map $\varphi: V_{x} \rightarrow \mathbf{R}^{2}$ as in Definition 3.2. If $V_{x}$ is sufficiently small, then the three components $A_{1}, A_{2}$ and $A_{3}$ of $V_{x} \backslash B R(W)$ are mapped by $\varphi$ homeomorphically into the plane and two of them, say $A_{1}$ and $A_{2}$, are mapped on the same side of $\varphi\left(V_{x} \cap B R(W)\right)$. We call a point in $A_{1} \cup A_{2}$ an arm point and a point in $A_{3}$ a stem point. Note that this definition does not depend on the choice of the map $\varphi$, provided that the regular neighborhood $V_{x}$ is sufficiently small. Furthermore, we call the set of the arm points the arm of $W$ with respect to $q$ and the set of the stem points the stem. Note that the arm and the stem satisfy a certain consistency condition in the neighborhoods of the non-simple points of $W$ (i.e., the points of $W$ which have conic neighborhoods as in Figure 1 (d)).

Let $W$ be a pseudo quotient map. Suppose that a structure of arm and stem around $B R(W)$ which satisfy the consistency condition at non-simple points is already given. Furthermore, we suppose that $W$ is oriented (see Definition 3.1). Then it is not difficult to see that the regular neighborhood of $\Sigma(W)$ can be topologically immersed into $\mathbf{R}^{2}$ so that the structure of arm and stem is respected.

Let $q: M \rightarrow W$ be a pseudo quotient map. If there exists a topological immersion $\eta: W \rightarrow \mathbf{R}^{2}$ which respects the structure of arm and stem of $W$ with respect to $q$, then we can find another topological immersion $\eta^{\prime}: W \rightarrow \mathbf{R}^{2}$ which is an approximation of $\eta$ and which is a generic smooth immersion with respect to $q$ : $M \rightarrow W$.

The purpose of this section is to prove the following.

Proposition 5.2. Let $W$ be a compact pseudo quotient space such that $H_{*}\left(W ; \mathbf{Z}_{2}\right) \cong H_{*}\left(\{\right.$ point $\left.\} ; \mathbf{Z}_{2}\right)$. We suppose that a structure of arm and stem which satisfies the consistency conditions at non-simple points is given to $W$. Furthermore, suppose that $W$ contains no cuspidal branch (Figure $1(\mathrm{c}-1)$ ) and no trident (Figure $1(\mathrm{~d}-5)$ ). Then there exists a topological immersion $\eta: W \rightarrow \mathbf{R}^{2}$ which respects the structure of arm and stem.

By using Proposition 5.2, we can prove Lemma 4.12 as follows.

Proof of Lemma 4.12. Firstly note that a structure of arm and stem it is already given to $W$, since we have a pseudo quotient map $q: M \rightarrow W$. Suppose that $W$ 


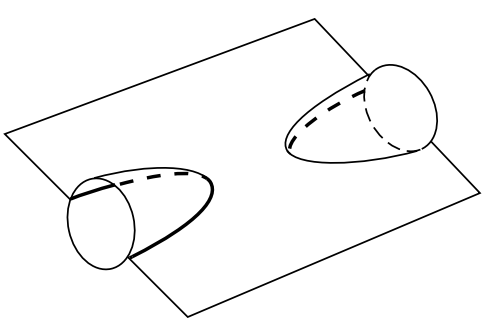

$\left(\mathrm{d}-1^{\prime}\right)$

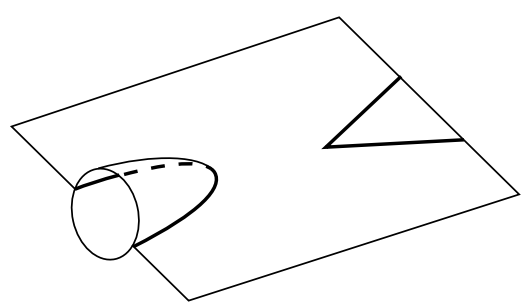

$\left(\mathrm{d}-4^{\prime}\right)$

FiguRE 5

contains no boundary point. Then $W$ contains no cuspidal branch. By Proposition 5.2 , there exists a topological immersion $\eta: W \rightarrow \mathbf{R}^{2}$. Since $W$ contains no boundary point, we easily see that $\eta$ is an open map. Thus $\eta(W)$ is a nonempty open compact set, which is a contradiction. \|

Proof of Proposition 5.2. First, for each point in $W$ which has a conic neighborhood as in Figure $1(\mathrm{~d}-1)$ or $(\mathrm{d}-4)$, replace the conic neighborhood with the space as in Figure $5\left(\mathrm{~d}-1^{\prime}\right)$ or $\left(\mathrm{d}-4^{\prime}\right)$ respectively. Denote by $W^{\prime}$ the resulting polyhedron. Then $W^{\prime}$ is a pseudo quotient space and inherits a structure of arm and stem induced from that of the original space $W$, since the original structure satisfies the consistency conditions at non-simple points. Note that $W^{\prime}$ is homotopy equivalent to $W$ and that, if $W^{\prime}$ is immersed into $\mathbf{R}^{2}$ respecting the structure of arm and stem, then so is $W$. Thus we may assume that each point of $W$ has a conic neighborhood as in Figure 1 (a) or (b). Since it has a structure of arm and stem, $W$ is a branched surface in the terminology of $[\mathbf{S a 2}]$. Then we can represent $W$ by its associated graph $G_{W}$ (for details, see $[\mathbf{S a 2}, \S 3]$ ). In the following, we use the terminology and notations introduced in $[\mathbf{S a 2}]$.

The following lemma is easy to prove by using the Mayer-Vietoris exact sequence of homology groups in $\mathbf{Z}_{2}$-coefficients.

Lemma 5.3. If $H_{1}\left(W ; \mathbf{Z}_{2}\right)=0$, then the associated graph $G_{W}$ is a tree.

Now we go back to the proof of Proposition 5.2. By the above lemma together with the assumption $H_{1}\left(W ; \mathbf{Z}_{2}\right)=0$, we see that $W$ is orientable. Then we see that, if $G_{W}$ has no double edge, $W$ can be immersed into $\mathbf{R}^{2}$ by [Sa2, Corollary 3.9]. (In [Sa2], $H_{2}(W ; \mathbf{Z})=0$ is assumed. However, the same argument works also under the assumption $H_{2}\left(W ; \mathbf{Z}_{2}\right)=0$.) We will prove the proposition by using the same argument as in the proof of Corollary 3.9 of [Sa2]. When the number $n$ of vertices of $G_{W}$ is greater than or equal to 3, we have to consider the forms as in Figure 6 (d), (e), (f) and (g) of $G_{W}$ other than the three forms (a), (b) and (c) of [Sa2, p. 90].

Suppose that $G_{W}$ is of the form (d). Let $R$ be the component of $W \backslash \Sigma(W)$ corresponding to the white vertex, where $\Sigma(W)$ is as defined in Definition 3.1 or in $[\mathbf{S a 2}]$. Then we see that there exists a non-zero element $\gamma \in H_{1}\left(R ; \mathbf{Z}_{2}\right)$ which cannot vanish in $H_{1}\left(W ; \mathbf{Z}_{2}\right)$. This is a contradiction. Thus the case (d) does not occur in our case. 
(d)

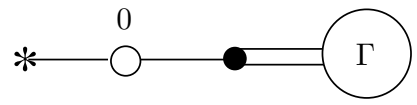

(e)

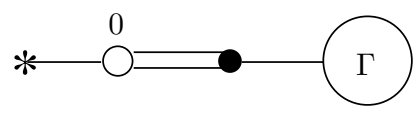

(f)

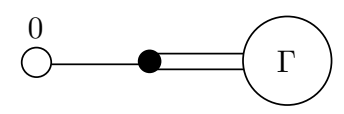

$(\mathrm{g})$

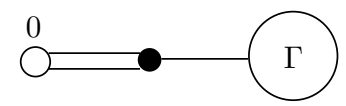

FiguRE 6

Suppose that $G_{W}$ is of the form (e). Then we see that the pseudo quotient space (or branched surface) whose associated graph is $\Gamma$ is homotopy equivalent to $W$ and hence that $\Gamma$ is appropriately directed as in [Sa2, Proposition 3.7] by the induction hypothesis. Thus $G_{W}$ is also appropriately directed.

Suppose that $G_{W}$ is of the form (f). Then we see that the pseudo quotient space whose associated graph is $\Gamma$ satisfies our homological hypothesis, by using an argument similar to the proof of Lemma 4.11. Then, by our induction hypothesis, $\Gamma$ is appropriately directed and hence $G_{W}$ is also appropriately directed.

Suppose that $G_{W}$ is of the form (g). Then we easily see that $H_{2}\left(W ; \mathbf{Z}_{2}\right) \neq 0$. This contradicts our assumption. Thus this case does not occur.

Thus, we have shown that $G_{W}$ is appropriately directed and hence that $W$ is immersed into $\mathbf{R}^{2}$ by [Sa2, Proposition 3.7]. This completes the proof. ॥

By using Definition 5.1, we can formulate the $R$-operation in the category of stable maps, not in the category of pseudo quotient maps, as follows.

Definition 5.4. Let $f: M \rightarrow \mathbf{R}^{2}$ be a stable map of a closed $n$-manifold $(n \geq 3)$ into the plane. We assume that $\pi_{0}\left(\operatorname{Diff}^{+}\left(S^{n-2}\right)\right)=0, n \geq 3, H_{1}(M ; \mathbf{Z})=0$, $H_{2}\left(W_{f} ; \mathbf{Z}\right)=0$ and that the pseudo quotient map $q^{\prime}: M \rightarrow W^{\prime}$ is obtained from $q_{f}: M \rightarrow W_{f}$ by an $R$-operation in the sense of Definition 4.5. Furthermore, we suppose that there exists a generic smooth immersion $\eta: W^{\prime} \rightarrow \mathbf{R}^{2}$ with respect to $q^{\prime}$. Then we say that the stable map $g=\eta \circ q^{\prime}: M \rightarrow \mathbf{R}^{2}$ is obtained from $f$ by an $R$-operation.

As a corollary to Theorem 4.13 and Proposition 5.2, we have the following.

Theorem 5.5. Let $f: M \rightarrow \mathbf{R}^{2}$ be a stable map of a closed connected n-manifold such that $\pi_{0}\left(\operatorname{Diff}^{+}\left(S^{n-2}\right)\right)=0, n \geq 4, \pi_{1}(M)=1, H_{2}\left(W_{f} ; \mathbf{Z}\right)=0$ and that $W_{f}$ contains no cuspidal branch (Figure $1(\mathrm{c}-1)$ ) and no trident (Figure $1(\mathrm{~d}-5)$ ). Then, 
by a finite iteration of $R$-operations performed on $f$, we can convert $f$ to a stable map $g: M \rightarrow \mathbf{R}^{2}$ such that $q_{g}: M \rightarrow W_{g}$ is configuration trivial with respect to the spherical regions.

The proof is easy and is left to the reader.

Remark 5.6. By the elimination theorem of cusp points by Levine [L1], every stable map $f: M \rightarrow \mathbf{R}^{2}$ of a closed orientable $n$-manifold $M(n \geq 3)$ with even Euler characteristic is homotopic to a stable map $g: M \rightarrow \mathbf{R}^{2}$ which has no cusp points. In particular, $W_{g}$ contains no cuspidal branch as in Figure 1 (c-1). In addition, by looking to the homotopy which was constructed by Levine, we see that we can arrange so that $W_{g}$ is homotopy equivalent to $W_{f}$. However, unfortunately, this homotopy may create a trident as in Figure $1(\mathrm{~d}-5)$.

We do not know whether, in Theorems 4.13 and 5.5, we can remove the condition on conic neighborhoods or not.

\section{4-DimENSIONAL CASES}

In this section, we concentrate on stable maps of 4-dimensional manifolds, and we give some applications of Theorems 4.13 and 5.5 to the simplification of certain stable maps from simply connected closed 4-manifolds into the plane. The main result Theorem 6.8 of this section is a refinement of Theorem B in [Kob1].

Definition 6.1. Let $M$ be a $C^{\infty} n$-manifold and $N$ a $C^{\infty}$ surface. A proper stable map $f: M \rightarrow N$ is called a stable fibration if each fiber $f^{-1}(a)(a \in N)$ is connected.

Note that if $f: M \rightarrow N$ is a stable fibration, its quotient space $W_{f}$ is always a topological manifold by Theorem 2.2 and Remark 2.3 .

Remark 6.2. Let $g: M \rightarrow N$ be a proper stable map of an $n$-manifold into a surface. Suppose that the associated quotient space $W_{g}$ is a topological manifold (with boundary). Then, we see that

(1) $\bar{g}: W_{g} \rightarrow N$ is a local homeomorphism, and we can give to $W_{g}$ a unique $C^{\infty}$-structure which makes $\bar{g}$ an immersion, and

(2) for a proper smooth embedding $h: W_{g} \rightarrow N^{\prime}$ to a smooth surface $N^{\prime}$ without boundary, the map $h \circ q_{g}: M \rightarrow N^{\prime}$ is a stable fibration.

In other words, studying a stable map into a surface whose quotient space is a topological manifold is equivalent to studying a stable fibration.

Definition 6.3. Let $f: M \rightarrow \mathbf{R}^{2}$ be a stable fibration of an $n$-manifold. A connected component $R$ of $\mathbf{R}^{2} \backslash C(f)$ is said to be a spherical region if the fiber $f^{-1}(a)$ over each point $a \in R$ is diffeomorphic to the $(n-2)$-sphere. Furthermore, we say that $f$ is configuration trivial with respect to the spherical regions if each bounded spherical region $R$ with $\bar{R} \cap f\left(S_{0}(f)\right) \neq \emptyset$ is homeomorphic to the open 2-disk. This is equivalent to the condition that the quotient map $q_{f}: M \rightarrow W_{f}$ is configuration trivial with respect to the spherical regions as a pseudo quotient map.

The following lemma states that if $M$ is simply connected, a stable fibration is in fact a map onto the 2-disk $D^{2}$. 
Lemma 6.4. Let $f: M \rightarrow \mathbf{R}^{2}$ be a stable fibration of a closed connected $n$ manifold. If $\pi_{1}(M)=1$, then the quotient space $W_{f}$ is homeomorphic to the 2-disk $D^{2}$.

Proof. Since $f$ is a stable fibration and $M$ is compact, $W_{f}$ is a compact surface with boundary. Furthermore, by Lemma 4.3 , we see that $\pi_{1}\left(W_{f}\right)=1$. Thus $W_{f}$ is homeomorphic to the 2-disk. $\|$

Proposition 6.5. Let $f: M \rightarrow \mathbf{R}^{2}$ be a stable fibration of a closed orientable $n$-manifold $(n \geq 4)$. Then there exists a homotopy $f_{t}: M \rightarrow \mathbf{R}^{2}(t \in[0,1])$ with $f_{0}=f$ such that

(1) each fiber of $f_{t}$ is connected,

(2) except for a finite number of $t$ 's, $f_{t}$ is a stable fibration,

(3) $W_{f_{1}}$ is homeomorphic to $W_{f}$, and

(4) $f_{1}$ is a stable fibration with at most one cusp point.

The above proposition is a consequence of Levine's theorem on elimination of cusp points $[\mathbf{L} \mathbf{1}]$. One can prove the above proposition by examining the homotopy given in $[\mathbf{L 1}]$ carefully. Recall that the number of cusp points has the same parity as the Euler characteristic of the source manifold. We should also note that, when we eliminate the extra cusp points, the homeomorphism types of the fibers may change.

In view of Proposition 6.5, we assume in the following that all stable fibrations have at most one cusp point.

Now we consider the 4-dimensional case. Suppose that $f: M \rightarrow \mathbf{R}^{2}$ is a stable fibration of a closed orientable 4-manifold. Note that, in this case, each fiber of $f$ is an orientable closed connected surface.

Definition 6.6. Let $f: M \rightarrow \mathbf{R}^{2}$ be a stable fibration of a closed orientable 4manifold with at most one cusp point. We say that $f$ is of torus type if each regular fiber of $f$ is homeomorphic either to the torus or to the sphere.

In what follows we deal with stable fibrations of torus type with at most one cusp point, of simply connected 4-manifolds into the plane, or onto a closed 2-disk by Lemma 6.4. Note that standard 4-manifolds such as $S^{4}, \mathbf{C} P^{2}, S^{2} \times S^{2}, S^{2} \tilde{\times} S^{2}$, and their connected sums admit stable fibrations as mentioned above, where $S^{2} \tilde{\times} S^{2}$ is the nontrivial $S^{2}$-bundle over $S^{2}$ (see [Kob1] for their constructions).

Lemma 6.7. Let $f: M \rightarrow \mathbf{R}^{2}$ be a stable fibration of torus type, where $M$ is an orientable closed 4-manifold. Then $f \mid S(f)$ is a topological embedding and $f \mid(S(f) \backslash\{$ cusp points $\})$ is a $C^{\infty}$ embedding.

Proof. Suppose that $f \mid S(f)$ is not a topological embedding. Then, by $[\mathbf{F u}]$ or $[\mathbf{P F}$, Theorem 1.13], we see that $f$ should have a fiber with genus greater than or equal to 2 , since the quotient space $W_{f}$ is a topological manifold. This is a contradiction. ॥

The following is a refinement of Kobayashi's result [Kob1] on simplifying stable fibrations of torus type.

Theorem 6.8. Let $M$ be a simply connected closed 4-manifold, and let $f: M \rightarrow \mathbf{R}^{2}$ be a stable fibration of torus type with at most one cusp point. Then, we have the following. 
(1) By a finite iteration of $R$-operations defined in $\S 5$, we can convert the map $f$ to a stable fibration $f^{\prime}: M \rightarrow \mathbf{R}^{2}$ of torus type with at most one cusp point which is configuration trivial with respect to the spherical regions, without changing the diffeomorphism type of the source manifold $M$.

(2) Besides the above operations, by performing a finite iteration of $C$-operations defined in [Kob1, §6] to $f^{\prime}$, we can convert $f$ to a stable fibration $h: M \rightarrow \mathbf{R}^{2}$ of torus type with at most one cusp point which is configuration trivial with respect to the spherical regions and which satisfies the following, without changing the diffeomorphism type of the source manifold $M$ :

$$
\begin{aligned}
& \left.\sharp S(h)=1 \quad \text { (if } b_{2}(M)=0\right), \\
& \sharp S(h) \leq \frac{3}{2} b_{2}(M)+1 \quad\left(\text { if } b_{2}(M) \text { is even and non-zero }\right), \\
& \sharp S(h) \leq \frac{3}{2}\left(b_{2}(M)+1\right) \quad\left(\text { if } b_{2}(M) \text { is odd }\right),
\end{aligned}
$$

where $\sharp S(h)$ denotes the number of connected components of $S(h)$ and $b_{2}(M)$ the second Betti number of $M$.

Remark 6.9. If we replace the words "R-operation" and "diffeomorphism" by "S-operation" and "homeomorphism" respectively, then the above result has been obtained in [Kob1, Theorem B]. Furthermore, by the same argument as in the proof of [Kob1, Theorem B (2)], we see that, in the above theorem, the pair $(M, h)$ has a decomposition

$$
(M, h)=\left(M_{1}, h_{1}\right) \natural\left(M_{2}, h_{2}\right) \natural \cdots \curvearrowleft\left(M_{k}, h_{k}\right),
$$

such that each map $h_{i}: M_{i} \rightarrow \mathbf{R}^{2}$ is a stable fibration with the same property as $h$ and that, in addition, $h_{i}$ has at most one connected component $R$ of $W_{h_{i}} \backslash q_{h_{i}}\left(S\left(h_{i}\right)\right)$ with $f^{-1}(a)(a \in R)$ homeomorphic to the torus (see Lemma 3.7). Here we have used the same notation as in [Kob1], although in the present paper, we have only defined connected sums of pseudo quotient maps.

Proof of Theorem 6.8. Firstly note that, by Lemma 6.4, the quotient space $W_{f}$ is homeomorphic to the 2-disk, which is contractible. Then we can apply Theorem 5.5 to obtain part (1). Part (2) is proved by the same argument as in [Kob1]. Note that, by Lemma 6.7, the maps here satisfy all the necessary conditions assumed in [Kob1]. \|

Corollary 6.10. Let $M$ be a simply connected closed 4-manifold with Euler characteristic $\chi(M)=2$. If $M$ admits a stable fibration of torus type $f: M \rightarrow \mathbf{R}^{2}$ without cusp points, then $M$ is diffeomorphic to $S^{4}$.

Proof. By Theorem 6.8, we see that there exists a stable fibration $h: M \rightarrow \mathbf{R}^{2}$ without cusp points such that $S(h)$ is made up of a single closed curve. Hence the curve must consist of definite fold points.

Then $W_{h} \backslash q_{h}\left(S_{0}(h)\right)$ is an open 2-disk by Lemma 6.4. This implies that $M$ is decomposed as $D^{2} \times S^{2} \cup S^{1} \times D^{3}$ according to the decomposition of $W_{h}$ into a collar neighbourhood $N(C)$ of $C=q_{h}\left(S_{0}(h)\right)$ in $W_{h}$ and $\overline{W_{h} \backslash N(C)}$. Then we see that $M$ is diffeomorphic to $S^{4}$, by using a result of Gluck [Gl] about diffeomorphisms of $\partial\left(S^{1} \times D^{3}\right)$. 
Alternatively, we can use a result of $[\mathbf{P F}]$, since $h$ is a special generic map. $\|$

We note that a simply connected closed 4-manifold $M$ with $\chi(M)=2$ is always homeomorphic to $S^{4}$ by $[\mathbf{F r}]$.

Let $f: M \rightarrow \mathbf{R}^{2}$ be a stable fibration of a closed orientable 4-manifold. In the following we say that a connected component $R$ of $\mathbf{R}^{2} \backslash C(f)$ is a 1-region if the fiber $f^{-1}(a)$ over each point $a$ of $R$ is homeomorphic to the torus, or the closed connected orientable surface of genus 1 .

Corollary 6.11. Let $f: M \rightarrow \mathbf{R}^{2}$ be a stable fibration of torus type with at most one cusp point of a closed simply connected 4-manifold $M$. Assume that $f$ is a trivial $T^{2}$-bundle when restricted over each 1-region. Then $M$ is diffeomorphic either to $\sharp^{k} S^{2} \times S^{2}$ or to $\sharp^{k} S^{2} \tilde{\times} S^{2}$ for some $k \geq 0$, where the connected sum over an empty set is assumed to be $S^{4}$.

Proof. By Theorem 6.8, we may assume that $f$ is configuration trivial with respect to the spherical regions. Note that, in the course of the $R$-operations performed on $f$, the triviality of the $T^{2}$-bundle over the 1-regions is preserved. Then the result follows from [Kob2, Theorem B] and Remark 6.9 of the present paper. II

We note that the 4-manifolds $\sharp^{k} S^{2} \times S^{2}$ and $\sharp^{k} S^{2} \tilde{\times} S^{2}$ admit such stable fibrations as in the above corollary ([Kob2] $)$.

Now we consider the Moishezon-Teicher surface $\tilde{X}_{7,4}$ ([MT1]), which is a simply connected compact complex surface of general type with zero signature. Since it is simply connected, has zero signature and is spin ([Kot1, Kot2]), it is homeomorphic to $\sharp^{k} S^{2} \times S^{2}$ for some $k$ by a result of Freedman [Fr], where $k$ is a high enough positive integer (see [MT2]). However, it is not diffeomorphic to $\sharp^{k} S^{2} \times S^{2}$ by a result of Donaldson $[\mathbf{D}]$. Thus we have the following.

Corollary 6.12. The Moishezon-Teicher surface $\tilde{X}_{7,4}$ is homeomorphic to $\sharp^{k} S^{2} \times S^{2}$ for some $k$, which admits such a stable fibration as in Corollary 6.11, but $\tilde{X}_{7,4}$ never admits such a stable fibration itself.

If we compare Corollary 6.12 with the examples mentioned just after Definition 6.6 , it seems to be an interesting problem to characterize those closed orientable 4-manifolds which admit stable fibrations of torus type with at most one cusp point. The following problem is left to a future investigation.

Problem 6.13. (1) Does there exist a closed simply connected 4-manifold which does not admit a stable fibration of torus type with at most one cusp point?

(2) Let $M$ be an arbitrary closed simply connected 4-manifold. Does there exist a smooth 4-manifold $N$ homeomorphic to $M$ which admits a stable fibration of torus type with at most one cusp point?

(3) Does the $K 3$ surface admit a stable fibration of torus type without cusp points?

We note that the answer for Problem 6.13 (2) is yes, provided $M$ is not spin.

\section{Pseudo QuOtient Maps With only SPherical Regular Fibers}

In this section, as an application of our simplification theorem, we consider pseudo quotient maps which have only spherical regions. The following theorem illustrates a typical example in which our simplification theorem is useful in determining the diffeomorphism type of the source manifold. 


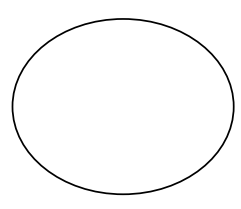

(a)

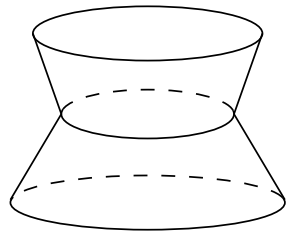

(b)

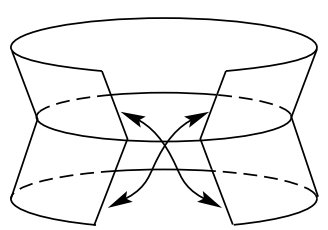

(c)

Figure 7

Theorem 7.1. Let $q: M \rightarrow W$ be a pseudo quotient map of a closed n-dimensional manifold $(n \geq 4)$ such that $n$ is even, $\pi_{1}(M)=1, H_{2}(W ; \mathbf{Z})=0$, q has no cusp points, and $W$ contains no trident. Suppose that every component of $W \backslash C(q)$ is a spherical region. Then $M$ is a homotopy $n$-sphere and, if $n \leq 6, M$ is diffeomorphic to the $n$-sphere.

Proof. First note that no point of $W$ has a conic neighborhood as in Figure 1 (b$3)$. This is seen as follows. If there exists such a point $x \in W$, then we see that there exists a compact $(n-1)$-dimensional manifold $X$ such that $\partial X$ consists of two $(n-2)$-spheres and that there exists a Morse function $h: X \rightarrow \mathbf{R}$ with exactly one critical point (consider the transverse manifold $X$ at $q^{-1}(x) \cap S(q)$ ). This is a contradiction, since $X$ is odd dimensional.

The above observation implies that every point of $W$ has a conic neighborhood as in Figure $1(\mathrm{a}),(\mathrm{b}-1)$ or $(\mathrm{b}-2)$, since $q$ has no cusp points and a conic neighborhood as in Figure 1 (d-1) (double cone) does not occur for $n \geq 4$ as remarked in the proof of Theorem 2.2.

First we prove the theorem when $\pi_{0}\left(\operatorname{Diff}^{+}\left(S^{n-2}\right)\right)=0$. By Theorem 4.13, we may assume that $q$ is configuration trivial with respect to the spherical regions. Furthermore, we may assume that each spherical region adjacent to $q\left(S_{0}(q)\right)$ is homeomorphic to the open annulus or to the open 2-disk by Lemma 3.7, since the connected sum of (homotopy) $n$-spheres is diffeomorphic to the $n$-sphere (or a homotopy $n$-sphere). On the other hand, the Euler characteristic of $W$ is equal to one, since it is contractible by Lemma 4.3. Together with the observation in the previous paragraph, this implies that $W$ has at most one region which is not adjacent to $q\left(S_{0}(q)\right)$ and that this region must be homeomorphic to the open 2disk. Furthermore, the other regions are homeomorphic to the open annulus or to the open 2-disk. Thus we see that $W$ is homeomorphic to one of the polyhedrons as in Figure 7 (a)-(c). Here, the polyhedron of Figure 7 (a) is homeomorphic to the 2-disk, that of (b) is homeomorphic to the union of the 2-disk $D^{2}$ and $Y \times S^{1}$ attached along $\partial D^{2}$ and $\{1\} \times S^{1}$, and that of (c) is homeomorphic to the union of the 2-disk $D^{2}$ and the twisted $Y$-bundle over $S^{1}$ with monodromy $\tau: Y \rightarrow Y$ attached along $\partial D^{2}$ and $\{1\} \times S^{1}$, where $Y$ is the subset of $\mathbf{C}$ defined just after Definition 5.1, and $\tau$ is the complex conjugation restricted to $Y$.

If $W$ is as in Figure 7 (a), then the result follows from [PF, Sa1], since there exists an embedding $\eta: W \rightarrow \mathbf{R}^{2}$ such that the map $f=\eta \circ q: M \rightarrow \mathbf{R}^{2}$ is a special generic map whose quotient space $W$ is contractible.

If $W$ is as in Figure 7 (b) or (c), let $N(\subset W)$ be a closed disk neighborhood of a point in the spherical region homeomorphic to the open 2-disk. Then it is not 
difficult to see that $q^{-1}(N)$ is diffeomorphic to $D^{2} \times S^{n-2}$ and that $q^{-1}(\overline{W \backslash N})$ is diffeomorphic to an orientable $D^{n-1}$-bundle over $S^{1}$. If $n=4$, then the latter is known to be diffeomorphic to $S^{1} \times D^{n-1}$. If $n \geq 6$, then we can show that every orientable $D^{n-1}$-bundle over $S^{1}$ is diffeomorphic to $S^{1} \times D^{n-1}$, by using the $s$-cobordism theorem. Then we see that $M=q^{-1}(N) \cup q^{-1}(\overline{W \backslash N})$ is a homotopy $n$-sphere.

When $\pi_{0}\left(\operatorname{Diff}^{+}\left(S^{n-2}\right)\right) \neq 0$, by using a similar argument in the $C^{0}$-category (see Remark 4.6), we see that $M$ is homeomorphic to the $n$-sphere and hence that $M$ is a homotopy $n$-sphere. This completes the proof. $\|$

Note that $S^{4}$ and every homotopy $n$-sphere $(n \geq 5)$ admits a pseudo quotient map as in Theorem 7.1 (see [Sa1]). Furthermore, we can construct such a pseudo quotient map which is as much complicated as we want. For this, we firstly construct a pseudo quotient map into a pseudo quotient space as in Figure 7 and apply the connected sum of some copies of these maps and then apply the converse of the $R$-operation as mentioned just after Definition 4.5. Note also that Theorem 7.1 can be regarded as a generalization of [PF, Proposition 2.6] and [Sa1, Proposition 4.1] concerning special generic maps with contractible quotient spaces.

Remark 7.2. Theorem 7.1 does not hold for $n$ odd in general. For example, we can construct a counterexample for $n=5$ as follows. Let $g: \mathbf{C} P^{2} \backslash \operatorname{Int} D^{4} \rightarrow[0,1]$ be a Morse function with one critical point $c_{0}$ of index 0 , one critical point $c_{2}$ of index 2 and no other critical points. We suppose that $g\left(c_{0}\right)=0, g\left(c_{2}\right)=1 / 2$ and $g^{-1}(1)=\partial\left(\mathbf{C} P^{2} \backslash \operatorname{Int} D^{4}\right)$. Define $q_{1}:\left(\mathbf{C} P^{2} \backslash \operatorname{Int} D^{4}\right) \times S^{1} \rightarrow[0,1] \times S^{1}$ by $q_{1}=g \times$ id. Furthermore, define $q_{2}: S^{3} \times D^{2} \rightarrow D^{2}$ to be the projection to the second factor. Then we set

$$
M=\left(\mathbf{C} P^{2} \backslash \operatorname{Int} D^{4}\right) \times S^{1} \cup S^{3} \times D^{2}
$$

and

$$
W=D^{2} \cup[0,1] \times S^{1}
$$

where $\partial D^{2}$ and $\{1\} \times S^{1}$ are identified. Note that $M$ is simply connected and that $W$ is homeomorphic to the 2-disk $D^{2}$. Finally we define $q: M \rightarrow W$ by $q(x)=q_{1}(x)$ for $x \in\left(\mathbf{C} P^{2} \backslash \operatorname{Int} D^{4}\right) \times S^{1}$ and $q(y)=q_{2}(y)$ for $y \in S^{3} \times D^{2}$. Note that $q$ is a pseudo quotient map such that every connected component of $W \backslash C(q)$ is a spherical region, that $q$ has no cusps, that $W$ contains no trident and that $W$ is contractible. However, we easily see that $H_{2}(M ; \mathbf{Z})$ is isomorphic to $\mathbf{Z}$, which shows that $M$ is not a homotopy 5 -sphere. Hence, Theorem 7.1 does not hold for $n=5$.

As an immediate corollary to Theorem 7.1, we have the following.

Corollary 7.3. Let $f: M \rightarrow \mathbf{R}^{2}$ be a stable map of a closed $n$-dimensional manifold $(n \geq 4)$ such that $n$ is even, $\pi_{1}(M)=1, H_{2}\left(W_{f} ; \mathbf{Z}\right)=0, f$ has no cusp points, and that $W_{f}$ contains no trident. Suppose that every component of $f^{-1}(a)$ is diffeomorphic to $S^{n-2}$ for all $a \in f(M) \backslash C(f)$. Then $M$ is a homotopy $n$-sphere and, if $n \leq 6, M$ is diffeomorphic to the $n$-sphere. 


\section{REFERENCES}

[BdR] O. Burlet and G. de Rham, Sur certaines applications génériques d'une variété close à 3 dimensions dans le plan, l'Enseign. Math. 20 (1974), 275-292. MR 51:1846

[C1] J. Cerf, Sur les difféomorphismes de la sphère de dimension trois $\left(\Gamma_{4}=0\right)$, Lecture Notes in Math. 53, Springer, Berlin, 1968. MR 37:4824

[C2] J. Cerf, La stratification naturelle des espaces de fonctions différentiables réelles et le théorème de la pseudo-isotopie, Publ. Math. Inst. Hautes Etudes Sci. 39 (1970), 5-173. MR 45:1176

[D] S. K. Donaldson, Polynomial invariants for smooth four-manifolds, Topology 29 (1990), 257-315. MR 92a:57035

[Fr] M. Freedman, The topology of four-dimensional manifolds, J. Diff. Geom 17 (1982), 357-453. MR 84b:57006

[Fu] Y. Furuya, Sobre aplicações genéricas $M^{4} \rightarrow \mathbf{R}^{2}$, Tese, ICMSC-USP, 1986.

[Gl] H. Gluck, The embeddings of two-spheres in the four-sphere, Trans. Amer. Math. Soc. 104 (1962), 308-333. MR 27:1924

[GG] M. Golubitsky and V. Guillemin, Stable mappings and their singularities, Grad. Texts in Math. 14, Springer, 1973. MR 49:6269

[H] A. E. Hatcher, A proof of the Smale conjecture, $\operatorname{Diff}\left(S^{3}\right) \simeq O(4)$, Ann. of Math. 117 (1983), 553-607. MR 85c:57008

[KM] M. Kervaire and J. Milnor, Groups of homotopy spheres: I, Ann. of Math. 77 (1963), 504-537. MR 26:5584

[Kob1] M. Kobayashi, Simplifying certain mappings from simply connected 4-manifolds into the plane, Tokyo J. Math. 15 (1992), 327-349. MR 93k:57062

[Kob2] M. Kobayashi, Stable maps with trivial monodromies and inactive log-transformations, preprint, Tokyo Institute of Technology, November 1993.

[Kot1] D. Kotschick, Orientation-reversing homeomorphism in surface geography, Math. Ann. 292 (1992), 375-381. MR 93f:14019

[Kot2] D. Kotschick, Non-trivial harmonic spinors on certain algebraic surfaces, Einstein metrics and Yang-Mills connections (ed. T. Mabuchi), Lecture Notes in Pure and Appl. Math., vol. 145, Dekker, New York, 1993, pp. 85-88. MR 94d:58138

[KLP] L. Kushner, H. Levine and P. Porto, Mapping three-manifolds into the plane I, Bol. Soc. Mat. Mexicana 29 (1984), 11-33. MR 86j:58011

[L1] H. Levine, Elimination of cusps, Topology 3 (suppl. 2) (1965), 263-296. MR 31:756

[L2] H. Levine, Classifying immersions into $\mathbf{R}^{4}$ over stable maps of 3-manifolds into $\mathbf{R}^{2}$, Lect. Notes in Math., vol. 1157, Springer-Verlag, Berlin-Heidelberg-New York-Tokyo, 1985. MR 88f:57056

[ML] L. E. Mata-Lorenzo, Polyhedrons and stable maps from 3-manifolds into the plane, unpublished note.

[Ma] J. Mather, Stability of $C^{\infty}$ mappings: VI. The nice dimensions, Proc. Liverpool Singularities - Symposium I, Lecture Notes in Math., vol. 192, Springer-Verlag, BerlinHeidelberg-New York, 1971, pp. 207-253. MR 45:2747

[Mi] J. Milnor, Lectures on the h-cobordism theorem, Princeton Univ. Press, Princeton, 1965. MR 32:8352

[MT1] B. Moishezon and M. Teicher, Existence of simply connected algebraic surfaces of general type with positive and zero indices, Proc. Nat. Acad. Sci. USA 83 (1986), 6665-6666. MR 87h:14034

[MT2] B. Moishezon and M. Teicher, Simply-connected algebraic surfaces of positive index, Inv. Math. 89 (1987), 601-643. MR 88f:14037

[MPS] W. Motta, P. Porto and O. Saeki, Stable maps of 3-manifolds into the plane and their quotient spaces, Proc. London Math. Soc. 71 (1995), 158-174.

[PF] P. Porto and Y. Furuya, On special generic maps from a closed manifold into the plane, Topology Appl. 35 (1990), 41-52. MR 91c:57034

[Sa1] O. Saeki, Topology of special generic maps of manifolds into Euclidean spaces, Topology Appl. 49 (1993), 265-293. MR 93m:57031 
[Sa2] O. Saeki, Simple stable maps of 3-manifolds into surfaces II, J. Fac. Sci. Univ. Tokyo 40 (1993), 73-124. MR 94k:57044

[Sm] S. Smale, Diffeomorphisms of the 2-sphere, Proc. Amer. Math. Soc. 10 (1959), 621-626. MR 22:3004

Department of Mathematics, Akita University, Akita 010, Japan

E-mail address: mahito@math.akita-u.ac.jp

Department of Mathematics, Faculty of Science, Hiroshima University, HigashiHiROSHIMA 739, JAPAN

E-mail address: saeki@top2.math.sci.hiroshima-u.ac.jp 